\title{
WestVirginiaUniversity
}

THE RESEARCH REPOSITORY @ WVU

Graduate Theses, Dissertations, and Problem Reports

2010

\section{Phase transition problems of conservation laws}

Chunguang Chen

West Virginia University

Follow this and additional works at: https://researchrepository.wvu.edu/etd

\section{Recommended Citation}

Chen, Chunguang, "Phase transition problems of conservation laws" (2010). Graduate Theses, Dissertations, and Problem Reports. 3115.

https://researchrepository.wvu.edu/etd/3115

This Dissertation is protected by copyright and/or related rights. It has been brought to you by the The Research Repository @ WVU with permission from the rights-holder(s). You are free to use this Dissertation in any way that is permitted by the copyright and related rights legislation that applies to your use. For other uses you must obtain permission from the rights-holder(s) directly, unless additional rights are indicated by a Creative Commons license in the record and/ or on the work itself. This Dissertation has been accepted for inclusion in WVU Graduate Theses, Dissertations, and Problem Reports collection by an authorized administrator of The Research Repository @ WVU.

For more information, please contact researchrepository@mail.wvu.edu. 


\title{
Phase Transition Problems of Conservation Laws
}

\author{
Chunguang Chen \\ Dissertation submitted to the \\ Eberly College of Arts and Sciences \\ at West Virginia University \\ in partial fulfillment of the requirements \\ for the degree of \\ Doctor of Philosophy \\ in \\ Mathematics \\ Harumi Hattori, Ph.D., Chair \\ Ismail Celik, Ph.D. \\ Mary Ann Clarke, Ph.D. \\ Harvey Diamond, Ph.D. \\ Dening Li , Ph.D. \\ Department of Mathematics \\ Morgantown, West Virginia \\ 2010
}

Keywords: Partial Differential Equation; Conservation Laws; Phase Transition

Copyright 2010 Chunguang Chen 


\section{Abstract \\ Phase Transition Problems of Conservation Laws \\ Chunguang Chen}

In this thesis we study phase transition problems of conservation laws. Phase transition problems arise from various applications such as gas dynamics, mechanics and material science. Conservation laws involving phase change is an attractive field in applied mathematics. Solutions to phase transition problems are complicated for the presence of boundaries between different phases. In addition to entropy condition, criteria such as kinetic relation $[\mathbf{1}, \mathbf{3}]$ and nucleation criterion are introduced to determine the configurations of solutions.

In Chapter1, we construct two numerical procedures to solve the Riemann problems for a system of conservation laws with phase change. We first find the solution with a stationary phase boundary by Newton iteration [14]. The configuration of the solution, especially the direction of the propagating phase boundary, is then determined based on the criterion suggested by Hattori [11] given that the speed of a moving phase boundary is much smaller than the speed of a shock or a rarefaction wave. One way to solve the Riemann problem with a moving phase boundary is to list all the relations and find the solution of the resulting nonlinear system. Another is to construct an iterative process to find the intersection of two projection curves.

In Chapter2, we discuss the well posedness of the initial value problem to Euler equations related to phase transition. The solution contains two phase boundaries moving in opposite directions. Entropy condition and kinetic relationship are used as the main admissibility criteria to select the physically relevant solution. We show the existence of the entropy solution under a suitable Finiteness Condition and a Stability Condition guarantees the stability of the problem in $L^{1} \cap B V$ and the existence of a Lipschitz semigroup of solutions. We also discuss the well posedness of the problem given that the wave speeds do not differ significantly between different phases. 


\section{Acknowledgments}

First, I would like to thank my adviser, Professor Harumi Hattori, for his help throughout this entire process. I have learned immensely from him and his guidance and encouragement made it possible to complete the Ph.D. thesis and the Ph.D. degree.

Secondly, I would like to thank my committee members, Professors Ismail Celik, Mary Ann Clarke, Harvey Diamond, Dening Li. Their advices throughout the process have been invaluable.

Thirdly, I would like to thank the Department of Mathematics at West Virginia University for providing me with an excellent study environment and support during my study as a graduate student.

Finally, I would like to thank my father, my mother, my sister, my wife and my daughter for their everlasting support without which this work could not have come out. 


\section{Contents}

List of Figures

List of Tables vii

Chapter 1. Numerical Solutions for Riemann Problems of Conservation Laws with Phase Change 1

1.1. Introduction

1.2. Waves in The Riemann Problem

1.3. Computation of Stationary Phase Boundary 6

1.4. Computation of Non-stationary Phase Boundary 7

1.5. Numerical Tests 12

Chapter 2. $L^{1}$ Well Posedness of Euler Equations With Dynamic Phase Boundaries 21

2.1. Introduction 21

2.2. Preliminaries 24

2.3. Front Tracking Approximations $\quad 27$

2.4. The Lyapunov Functional and Stability $\quad 35$

2.5. Stability of Approximate Solution 38

$\begin{array}{ll}\text { Bibliography } & 48\end{array}$ 


\section{List of Figures}

1.2.1 Configuration of solution with a stationary phase boundary.

1.2.2 Configuration of the solution to a Riemann problem with a forward phase boundary (FPB).

1.2.3 Configuration of the solution to a Riemann problem with a backward phase boundary (BPB).

1.3.1 The projections of 1 and 3 shock-rarefaction curves onto the $\sigma v$-plane. The solution of the Riemann problem with two shocks and a stationary phase boundary.

1.4.1 The definition of critical entropy $s^{m}$.

1.4.2 The Exact Riemann Solver II: Iterative Method.

1.5.1 $\sigma v$-projections of the shock-rarefaction curves.

1.5.2 $\sigma v$-projections of the shock-rarefaction curves.

1.5.3 (Upper)The intersection point $\left(\sigma^{*}, v^{*}\right)$ of the $\sigma v$-projections of the shockrarefaction curves. (Lower)The $\sigma v$-projections of the shock-rarefaction curves and the backward phase boundary curves.

1.5.4 (Upper)The intersection point $\left(\sigma^{*}, v^{*}\right)$ of the $\sigma v$-projections of the shockrarefaction curves. (Lower)The $\sigma v$-projections of the shock-rarefaction curves and the forward phase boundary curves.

1.5.5 (Upper)The intersection point $\left(\sigma^{*}, v^{*}\right)$ of the $\sigma v$-projections of the shockrarefaction curves. (Lower)The $\sigma v$-projections of the shock-rarefaction curves and the forward phase boundary curves.

2.1.1 The solution to the initial value problem (2.1.1) (2.1.3). The upper $x t$-plane is divided into three regions $\Omega^{-}, \Omega^{m}$ and $\Omega^{+}$by the phase boundaries $P_{1}$ and $P_{2}$.

2.1.2 An example of a level curve $f(v, s)=c$.

2.2.1 The definition of $m_{13}^{P_{1}}$ (a) and $m_{31}^{P_{2}}$ (b).

2.3.1 Approximate Riemann Solver.

2.5.1 Case 1 38

2.5.2 Case 2.

40

2.5.3 The definition of $\hat{V}$.

2.5.4 The wave interaction pattern $\left(U^{-}, \hat{V}\right)+(\hat{V}, V) \longrightarrow\left(U^{-}, V\right) . \quad 42$

2.5.5 Case 3

2.5.6 Case 4

43 
2.5.7 Case A

2.5.8 Case B

46

2.5.9 Case C

47

2.5.10Case D 


\section{List of Tables}

1 Constants. 12

2 Initial value of Riemann problem. 12

3 The entropies. 13

4 Solution with a stationary phase boundary (SPB). 13

$5 \quad$ Initial value of Riemann problem. $\quad 13$

6 The entropies. 14

$7 \quad$ Solution with a stationary phase boundary (SPB). 14

8 Initial value of Riemann problem. 14

9 Solution by assuming that the phase boundary is stationary. 14

10 The entropies. 14

11 Solution with a backward phase boundary. 15

12 Initial value of Riemann problem. 15

13 Solution by assuming that the phase boundary is stationary. 15

14 The entropies. 15

15 Solution with a forward phase boundary. 18

16 Initial value of Riemann problem. 18

17 Solution by assuming that the phase boundary is stationary. 18

18 The entropies. 18

19 Solution with a forward phase boundary. 20 


\section{CHAPTER 1}

\section{Numerical Solutions for Riemann Problems of Conservation Laws with Phase Change}

\subsection{Introduction}

Numerical simulation of phase transition problems of conservation laws is currently an active field in applied mathematics. Solving Riemann problems plays an essential role in Godunov-type methods in which the initial value is approximated by a step function and a Riemann problem arises at each discontinuity. In this chapter, we investigate a mathematical model of martensitic phase transitions [21] between a low-strain phase ( $\alpha$-phase) and a high-strain phase ( $\beta$-phase). This model is used to study the dynamics of phase boundaries in elastic solids, for example, the shape memory alloys.

We consider a longitudinal deformation of homogeneous bar with unit cross section. Let $u(x, t)$ represent displacement of a reference point $x$ at $t, w=u_{x}$ is strain and $v=u_{t}$ is the particle velocity. The standard balance of mass, linear momentum and energy for adiabatic motions take the form

$$
\left\{\begin{array}{c}
w_{t}-v_{x}=0, \\
v_{t}-\sigma_{x}=0, \\
E_{t}-(\sigma v)_{x}=0,
\end{array}\right.
$$

where $t \geq 0,-\infty<x<\infty . \sigma$ and $E$ are stress and total energy, respectively. $E=e+u^{2} / 2$ where $e(w, s)$ is the specific internal energy ( $s$ is the specific entropy) and

$$
\sigma=e_{w}(w, s)
$$

When $\sigma_{w}(w, s)>0$, the system (1.1.1) is hyperbolic with three characteristic speeds

$$
\lambda=\left\{-\sqrt{\sigma_{w}}, 0, \sqrt{\sigma_{w}}\right\} .
$$

The solution of the Riemann problem contains a backward wave, a contact discontinuity and a forward wave corresponding to the three characteristic families when no phase transition occurs. In order to pick up the physically relevant solution, we need the entropy condition which imposes that the physical entropy increases across jump discontinuity. Let

$$
F=D\left(s_{+}-s_{-}\right),
$$

where $D= \pm \sqrt{\frac{\sigma_{+}-\sigma_{-}}{v_{+}-v_{-}}}$is the speed of jump discontinuity and the subscripts - and + denote the states to the left and right of discontinuity respectively. The entropy condition requires $F \leq 0$ holds across each discontinuity.

At the presence of phase change, one or more phase boundaries must be included in the Riemann solutions. The kinetic relation proposed by Abeyaratne and 
Knowles $[\mathbf{2}, \mathbf{4}]$ is used to determine uniquely the solution involving a propagating phase boundary. It postulates that there exists a non-decreasing function $\phi(g)$ satisfying $\phi(0)=0$, where $g$ is called the driving traction, such that the speed of phase boundary is given by

$$
D_{p}=\phi(g) .
$$

In order that this relation is consistent with the entropy condition, we require that $\phi^{\prime}>0$ so that $\sigma g>0$ holds. In this paper we choose $g=-\left(s_{+}-s_{-}\right)$for the driving traction. In particular, we use the following kinetic condition

$$
D_{p}=\epsilon\left(s_{-}-s_{+}\right),
$$

where $\epsilon$ is a small positive constant. This relation is applied to the solutions satisfying the entropy condition.

We consider the following stress-strain relation [21]

$$
\sigma(w, T)=A+B T+K\left(w-w_{a}\right)\left(w-w_{b}\right)\left(w-\frac{1}{2}\left(w_{b}+w_{a}\right)\right),
$$

where $\mathrm{A}, \mathrm{B}$ and $\mathrm{K}$ are positive constants. We assume that the specific heat $c$ is constant and the Helmholtz free energy can be written as

$$
f(w, T)=A w+B T w+K \psi(w)-c T \ln \frac{T}{T_{0}},
$$

where

$$
\psi(w)=\frac{w^{4}}{4}-\frac{1}{2}\left(w_{a}+w_{b}\right) w^{3}+\frac{1}{2} w^{2}\left(\frac{w_{a}^{2}}{2}+2 w_{a} w_{b}+\frac{w_{b}^{2}}{2}\right)-w_{a} w_{b}\left(\frac{w_{a}+w_{b}}{2}\right) w
$$

and

$$
\psi^{\prime}(w)=\left(w-w_{a}\right)\left(w-w_{b}\right)\left(w-\frac{1}{2}\left(w_{b}+w_{a}\right)\right) .
$$

The entropy is given by

$$
s(w, T)=-B w+c \ln \frac{T}{T_{0}}+c .
$$

Solve for $T$ to obtain that

$$
T=T_{0} \exp \left(\frac{s+B w-c}{c}\right)
$$

Plugging $T$ into $\sigma(w, T)$ gives

$$
\sigma(w, s)=A+B T_{0} \exp \left(\frac{s+B w-c}{c}\right)+K\left(w-w_{a}\right)\left(w-w_{b}\right)\left(w-\frac{1}{2}\left(w_{b}+w_{a}\right)\right) .
$$

By setting $\sigma(w, s)=\sigma_{0}$, we have the the entropy-strain relation at constant stress as

$$
s=-B w+c\left\{1+\ln \frac{1}{B T_{0}}\left[\sigma_{0}-A-K\left(w-w_{a}\right)\left(w-w_{b}\right)\left(w-\frac{1}{2}\left(w_{b}+w_{a}\right)\right)\right]\right\} .
$$

The internal energy is given by

$$
\begin{aligned}
e & =f+T s \\
& =A w+K \psi(w)+c T_{0} \exp \left(\frac{s+B w-c}{c}\right) .
\end{aligned}
$$




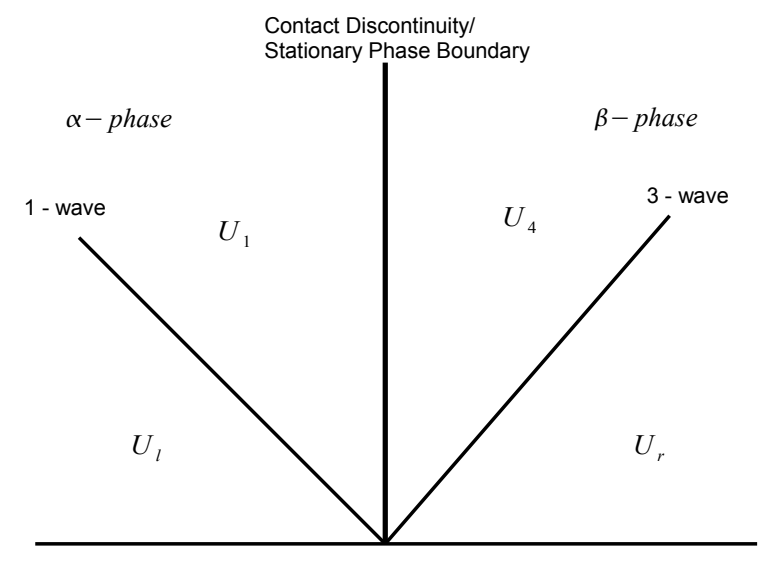

Figure 1.2.1. Configuration of solution with a stationary phase boundary.

If we express the internal energy e, stress $\sigma$ and temperature $T$ as functions of $w$ and $s$, it is easy to see that they satisfy the thermodynamic relation

$$
d e=\sigma d w+T d s,
$$

i.e. $\sigma=e_{w}$ and $T=e_{s}$.

\subsection{Waves in The Riemann Problem}

Denote by $U=[w, v, \sigma]^{T}$ the solution vector of (1.1.1). In the Riemann problem we seek a self-similar solution where $U_{l}$, the middle constant states, and $U_{r}$ are separated by the backward and forward waves that are shock or rarefaction waves, phase boundary and contact discontinuity or stationary phase boundary. These waves and contact discontinuity are well studied so that we briefly describe them and examine the phase boundary in details. The configurations of the Riemann solutions are shown in Fig1.2.1, Fig1.2.2 and Fig1.2.3, where $U_{l}$ and $U_{r}$ are specified in low-strain state ( $\alpha$-phase) and high-strain state ( $\beta$-phase), respectively.

\section{Rarefaction wave}

This is a continuous solution $U(\xi)$ of (1.1.1) with $\xi=x / t$. The rarefaction curve $R\left(U_{0}\right)$ is the set of $U$ connected to $U_{0}$ by a rarefaction wave. This set satisfies the differential equation

$$
\frac{d U}{d \xi}=\gamma_{k} \quad k=b, f
$$

where $\gamma_{k}(k=b, f)$ are given by

$$
\begin{gathered}
\gamma_{b}=\left(1, \sqrt{\sigma_{w}}, 0\right)^{T}, \\
\gamma_{f}=\left(1,-\sqrt{\sigma_{w}}, 0\right)^{T} .
\end{gathered}
$$




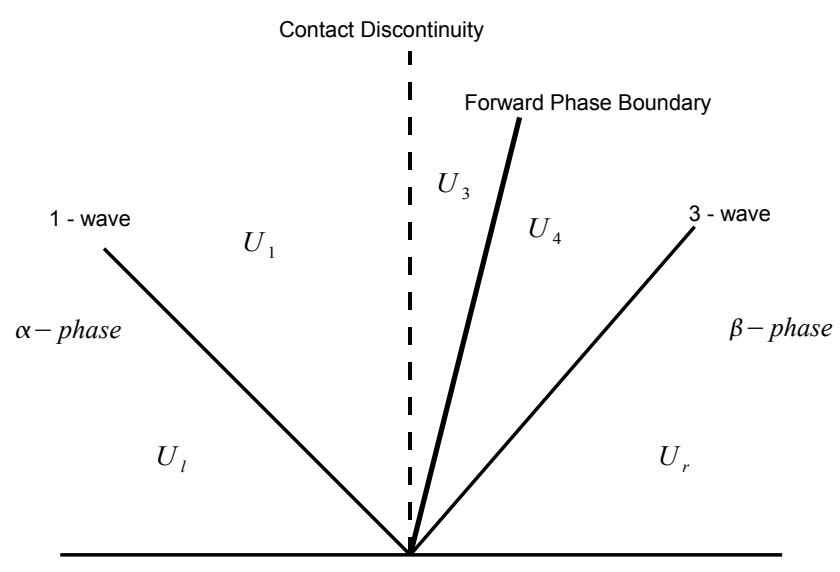

Figure 1.2.2. Configuration of the solution to a Riemann problem with a forward phase boundary (FPB).

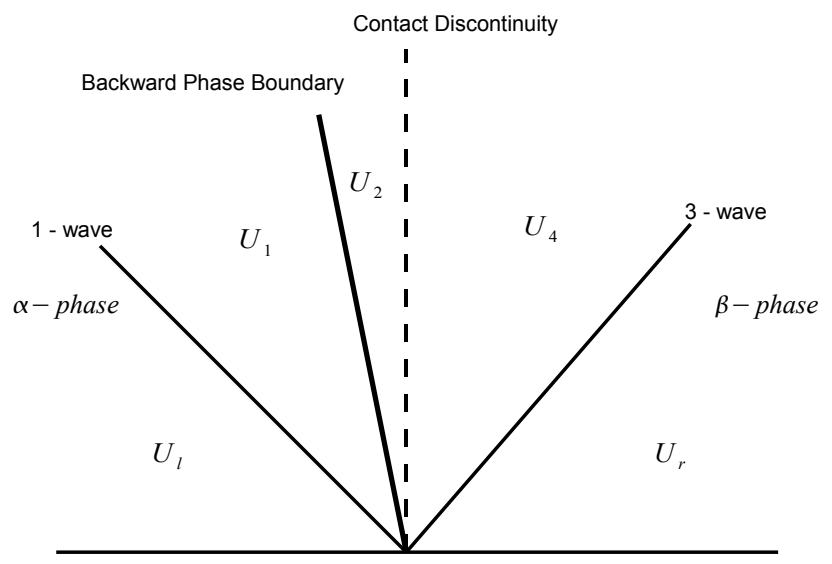

Figure 1.2.3. Configuration of the solution to a Riemann problem with a backward phase boundary (BPB).

\section{Shock wave}

This is a line of discontinuity in the $x-t$ plane. The shock curve $S\left(U_{0}\right)$ is the set of $U$ connected to $U_{0}$ by a shock wave. The two states satisfy the Rankine-Hugoniot condition given by 


$$
\begin{gathered}
D\left(w-w_{0}\right)=-\left(v-v_{0}\right), \\
D\left(v-v_{0}\right)=-\left(\sigma-\sigma_{0}\right), \\
D\left(E-E_{0}\right)=-\left(\sigma v-\sigma_{0} v_{0}\right),
\end{gathered}
$$

where $D$ is the speed of the shock. Usually, $U$ is the state on the right of $U_{0}$ connected by a rarefaction wave or a shock. In this paper $U$ is connected to $U_{0}$ either on the left or right of $U_{0}$. Obviously, the entropy condition should be satisfied.

We can verify that

$$
\begin{aligned}
\frac{\partial \sigma}{\partial w}= & \frac{B^{2} T_{0}}{c} \exp \left(\frac{s+B w-c}{c}\right) \\
& +K\left[3 w^{2}-3\left(w_{a}+w_{b}\right) w+\frac{1}{2}\left(w_{a}^{2}+w_{b}^{2}\right)+2 w_{a} w_{b}\right]>0
\end{aligned}
$$

except for the unobservable state. We can also show that with the specified constants in our numerical tests, $w$ increases across a shock in high-strain state, i.e., shock waves are decompressive in high-strain state. In the low-strain state, $w$ decreases across the shocks, i.e., shocks in low-strain state are compressive. For example, in Fig1.2.1, the 1-wave is a shock if $w_{1}<w_{l}\left(\sigma_{1}<\sigma_{l}\right)$ and is a rarefaction wave otherwise because $U_{l}$ and $U_{1}$ are in low-strain phase. The 3 -wave is a shock if $w_{4}>w_{r}\left(\sigma_{4}>\sigma_{r}\right)$ and is a rarefaction wave otherwise as $U_{r}$ and $U_{4}$ are in high-strain phase.

\section{Contact discontinuity and stationary phase boundary}

These are jump discontinuities across which the relations

$$
\sigma=\sigma_{0}, v=v_{0}
$$

hold. In Lagrangian coordinates, the contact discontinuity happens along a vertical line in $x t$-plane. If the phase changes across discontinuity, it is called a stationary phase boundary.

\section{Phase boundary}

A phase boundary is a line of discontinuity in $x t$-plane across which phase changes. It satisfies the Rankine-Hugoniot condition as well as the kinetic relation. In this paper, we define a forward phase boundary curves and backward phase boundary curve. In the configuration of solutions in Fig1.2.2, for example, $U_{3}$ is a state connected to $U_{4}$ through a forward phase boundary and $U_{4}$ to $U_{r}$ through a 3wave (forward shock or rarefaction wave). For a given state $U_{r}$, the set of all possible states of $U_{4}$ forms a one-parameter family of solutions whose graph is a curve in the three dimensional phase space and is called a forward shock-rarefaction curve. For any state $U_{4}$, the admissible state $U_{3}$ is uniquely determined by the following $4 \times 4$ system:

$$
\begin{gathered}
D_{p}\left(w_{4}-w_{3}\right)=-\left(v_{4}-v_{3}\right), \\
D_{p}\left(v_{4}-v_{3}\right)=-\left(\sigma_{4}-\sigma_{3}\right), \\
D_{p}\left(E_{4}-E_{3}\right)=-\left(\sigma_{4} v_{4}-\sigma_{3} v_{3}\right), \\
D_{p}=\epsilon\left(s_{3}-s_{4}\right),
\end{gathered}
$$


where $w_{3}, v_{3}, \sigma_{3}, D_{p}$ are unknown variables and $E_{3}, s_{3}$ are functions of $U_{3}=$ $\left(w_{3}, v_{3}, \sigma_{3}\right)^{T}$. Since one state of $U_{4}$ determines a unique state of $U_{3}$, the set of $U_{4}$ determines a set of $U_{3}$ whose graph is also a curve in the phase space of $(w, v, \sigma)$. This curve, denoted by $P_{f}\left(U_{r}\right)$, is called a forward phase boundary curve.

Similarly, if we consider the solution configuration in Fig1.2.3, we can define a backward phase boundary curve, denoted by $P_{b}\left(U_{l}\right)$, which is the set of all states of $U_{2}$.

\subsection{Computation of Stationary Phase Boundary}

In this and the following sections, we assume that the initial states are specified in different phases denoted by $\alpha$ and $\beta$, or low-strain state and high-strain state, respectively. We first compute the Riemann problem with a stationary phase boundary (SPB). Other cases with moving phase boundaries will be treated as perturbations of this case given the phase boundary moves slowly relative to a shock or rarefaction wave.

The intermediate constant states of a Riemann problem with a stationary phase boundary are depicted in Fig1.2.1 where $U_{i}=\left(w_{i}, v_{i}, \sigma_{i}\right)^{T} i=l, r, 1,4$ represent the constant states. We use numbers 1 and 4 to make it consistent with non-stationary cases where we have more intermediate constant states.

In this case, $U_{l}$ and $U_{r}$ are given initial states. $U_{1}$ and $U_{4}$ are connected by contact discontinuity or SPB. Thus,

$$
\sigma_{1}=\sigma_{4}, v_{1}=v_{4}
$$

A shock curve is the set of all possible states connected to the given state by a shock. Multiplying (1.2.2) by $\left(v+v_{0}\right) / 2$, subtracting it from (1.2.3) and using (1.2.1) give

$$
e-e_{0}=\frac{1}{2}\left(\sigma+\sigma_{0}\right)\left(w-w_{0}\right),
$$

where $e$ is given by (1.1.4). Moreover, dividing (1.2.3) by (1.2.2) gives

$$
\left(\sigma-\sigma_{0}\right)\left(e-e_{0}\right)=\frac{1}{2}\left(\sigma+\sigma_{0}\right)\left(v_{0}-v\right)^{2} .
$$

We have from (1.2.1) and (1.2.2) that

$$
\left(v-v_{0}\right)^{2}=\left(\sigma-\sigma_{0}\right)\left(w-w_{0}\right) .
$$

Combining (1.3.3) and (1.3.4) gives

$$
v=v_{0} \pm\left[\left(\sigma-\sigma_{0}\right)\left(w-w_{0}\right)\right]^{1 / 2},
$$

where \pm signs are taken for forward and backward waves, respectively. The shock curve is defined by (1.3.2) and (1.3.5).

Similarly, a rarefaction curve is the set of all possible states connected to a given state by a rarefaction wave. The rarefaction curve can be derived from Riemann invariants that are constants across the rarefaction wave, that is,

$$
\begin{gathered}
s=\text { constant } \\
v \pm \int^{w}\left(\sigma_{w}(w, s)\right)^{1 / 2}=\text { constant }
\end{gathered}
$$

where $s$ is the entropy. Therefore, the rarefaction curve is determined by

$$
s=s_{0},
$$




$$
v=v_{0} \mp \int^{w}\left(\sigma_{w}(w, s)\right)^{1 / 2},
$$

where we take "-/+" for a forward/backward rarefaction wave, respectively.

The solution for the Riemann problem with a stationary phase boundary contains a backward wave, a contact discontinuity (or SPB) and a forward wave. The backward and forward waves are shock and rarefaction waves. We can find the solution through an iterative method $([\mathbf{1 4}])$ as follows. Given the initial states $U_{l}$ and $U_{r}$, in order to find $U_{1}$ and $U_{4}$, we project the forward wave curve (FWC) and the backward wave curve (BWC) onto the $\sigma v$-plane and denote the them by $v=P_{4}(\sigma)$ and $v=P_{1}(\sigma)$, respectively. The projection curves intersect with each other at some points $\left(\sigma^{*}, v^{*}\right)$. We see from (1.3.1) that

$$
\begin{gathered}
\sigma_{1}=\sigma_{4}=\sigma^{*}, \\
v_{1}=v_{4}=v^{*} .
\end{gathered}
$$

$w_{i}(i=1,4)$ can be found by (1.3.4) or (1.3.6) given the $i$-wave is a shock or rarefaction wave.

\section{Computation of the projection of the shock curve}

The shock curve is defined by (1.3.2) and (1.3.5). In order to depict the projection of this curve onto the $\sigma v$-plane, we need to compute for each $\sigma$ value in its domain the corresponding $v$ value. This can be done by the following steps:

Step1: Given $\sigma$, solve (1.3.2) for $w$. We need an iteration, for example, Newton iteration, to solve this nonlinear equation.

Step2: Plug $w$ into (1.3.5) to compute $v$.

2. Computation of the projection of the rarefaction curve

Similarly, we use the following steps to find the rarefaction curve which is defined by (1.3.6) and (1.3.7):

Step1: Given $\sigma$, solve (1.3.6) for $w$ through iteration.

Step2: Substitute $w$ into (1.3.7) to compute $v$.

As long as we have the projections of shock and rarefaction curves connected to $U_{l}$ and $U_{r}$, we can find the intersection point $\left(\sigma^{*}, v^{*}\right)$ on the $\sigma v$ - plane (see Fig1.3.1).

Moreover, we can find the configuration of the solution with a stationary phase boundary. In the example in Fig1.3.1, both forward and backward waves are shocks since $\sigma_{r}<\sigma^{*}<\sigma_{l}$ (see section1.2.2).

\subsection{Computation of Non-stationary Phase Boundary}

The cases of moving phase boundaries are more complicated than stationary case for we have more intermediate constant states. We consider the case where the speed of a moving phase boundary is slow compared with that of the shock or rarefaction waves such that the configurations of 1 and 3-wave remain the same for any change of the phase boundary speed. This case is important in physics and we treat the dynamic phase boundary as a perturbation of a stationary one.

The first thing we need to know is how we can decide whether a phase boundary is stationary or not. If it is non-stationary, is it moving forward or backward? To answer this question, we introduce the following lemma ([11]). 


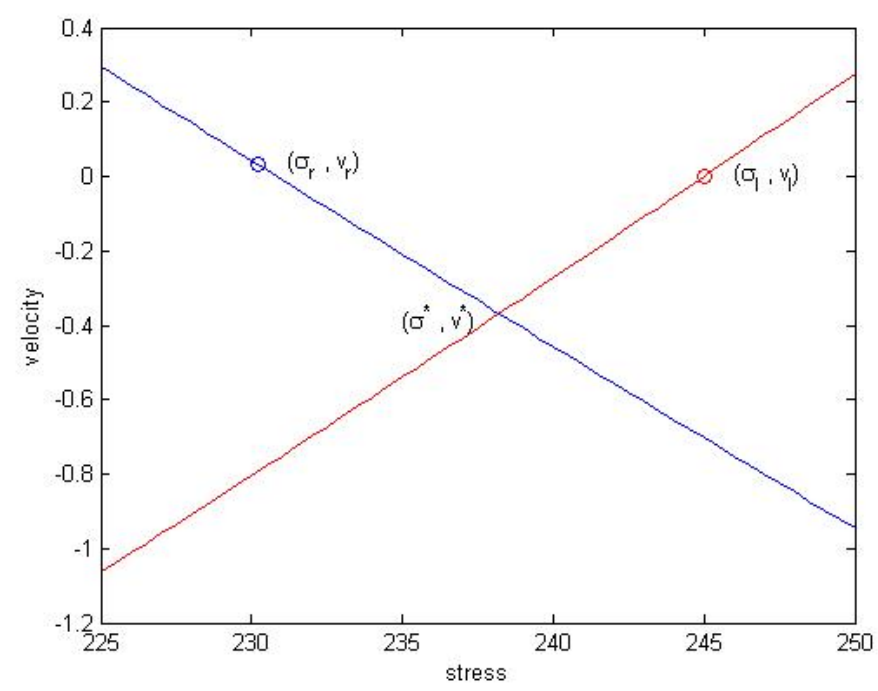

Figure 1.3.1. The projections of 1 and 3 shock-rarefaction curves onto the $\sigma v$-plane. The solution of the Riemann problem with two shocks and a stationary phase boundary.

Lemma 4.1 In the ws-plane, if the level curve $\sigma=\sigma_{0}$ is not monotone, there exists a unique state $\left(w_{0}^{m}, s_{0}^{m}\right)$ on $\sigma=\sigma_{0}$ with $w_{0}^{m}$ in the $\alpha$-phase such that the phase boundary emanates from $\left(w_{1}^{m}, s_{0}^{m}\right)$ on the same level curve with $w_{1}^{m}$ in the $\beta$-phase, i.e., the entropies have the same values (Fig1.4.1).

$s_{0}^{m}$ is called the critical entropy that will be used to determine the solution configurations. To calculate $s_{0}^{m}$ in the case of stationary phase boundary, we make use of the fact that (1.3.1) holds across the SPB. Let $U_{0}^{*}$ be the state connected to $U_{0}$ by a phase boundary. Therefore, $U_{0}^{*}$ and $U_{0}$ lie on the same $w s-$ plane, and on the same curve

$$
\sigma(v, s)=\sigma_{0} .
$$

Setting $\sigma=\sigma_{0}$ in (1.3.2) gives

$$
e-e_{0}=\sigma_{0}\left(w-w_{0}\right),
$$

especially,

$$
e_{0}^{*}-e_{0}=\sigma_{0}\left(v_{0}^{*}-v_{0}\right) .
$$

Combining this equation with

$$
s_{0}=s_{0}^{*}
$$

gives a system of $w_{0}$ and $w_{0}^{*}$. Solving this system gives us the solutions for $w_{0}$ and $w_{0}^{*}$ and the critical entropy can be found as $s_{0}^{m}=s_{0}$.

If $w_{l}$ and $w_{r}$ are specified in different phases we have the following results of solution configurations [11]. 


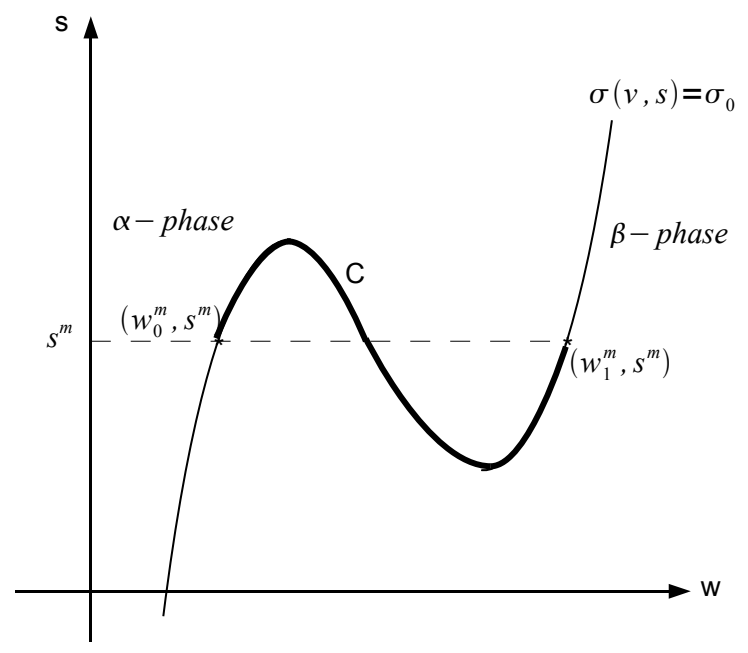

Figure 1.4.1. The definition of critical entropy $s^{m}$.

Theorem 4.2 If $w_{l}$ and $w_{r}$ are specified in different phases, there are four different solution configurations near $\sigma_{p}=0$ depending on the values of $s_{1}$ and $s_{4}$ at $D_{p}=0$.

(1) If $s_{1} \geq s^{m}$ and $s_{4} \leq s^{m}$ at $D_{p}=0$, then the solution with the stationary phase boundary is the only solution satisfying the entropy condition.

(2) If $s_{1}<s^{m}$ and $s_{4} \leq s^{m}$ at $D_{p}=0$, there is a one-parameter family of solutions with the backward phase boundary.

(3) If $s_{1} \geq s^{m}$ and $s_{4}>s^{m}$ at $D_{p}=0$, there is a one-parameter family of solutions with the forward phase boundary.

(4) If $s_{1}<s^{m}$ and $s_{4}>s^{m}$ at $D_{p}=0$, there are three solution configurations; there are two one-parameter families of solutions, one with the backward phase boundary and another with forward phase boundary. Also it is possible to construct the solutions with three phase boundaries where the left phase boundary moves backward, the middle one is stationary and the right one moves froward. In this case we have a two-parameter family of solutions.

1.4.1. Exact Riemann Solver (I): Nonlinear System. The solution of a Riemann problem can be found as follows:

Step1: Given any Riemann problem where $U_{l}$ and $U_{r}$ are specified in different phases, we can find the two intermediate states $U_{1}$ and $U_{4}$ with the process introduced in Section1.3.

Step2: Compute $s^{m}, s_{1}$ and $s_{4}$ with $U_{1}$ and $U_{4}$ and decide the configuration of the phase boundary by Theorem $\mathbf{4 . 2}$. 
Step3: If (1) in Theorem $\mathbf{4 . 2}$ is satisfied, we have a stationary phase boundary and the computation is done. If (1) is not satisfied, we have one or more moving phase boundaries. For example, when (3) is satisfied, the phase boundary moves forward (Fig1.2.2) and the solution contains 3 unknown constant states denoted by $U_{1}, U_{3}$ and $U_{4}$, respectively. The speed of the phase boundary satisfies the kinetic relation (2.1.4). We take $\epsilon=0.1$ in our numerical analysis.

If our solution in Step1 contains a backward shock and a forward rarefaction wave, (the configurations of the forward and backward waves are determined in Step1, and since $D_{p}$ is very small, they do not change when a non-stationary phase boundary is introduced) then we have the following equations:

(1) Backward shock wave:

$$
\begin{gathered}
D_{1}\left(w_{1}-w_{l}\right)=-\left(v_{1}-v_{l}\right), \\
D_{1}\left(v_{1}-v_{l}\right)=-\left(\sigma_{1}-\sigma_{l}\right), \\
D_{1}\left(E_{1}-E_{l}\right)=-\left(\sigma_{1} v_{1}-\sigma_{l} v_{l}\right) .
\end{gathered}
$$

(2) Contact discontinuity:

$$
\begin{aligned}
& \sigma_{1}=\sigma_{3}, \\
& v_{1}=v_{3} .
\end{aligned}
$$

(3) Forward phase boundary:

$$
\begin{gathered}
D_{p}\left(w_{4}-w_{3}\right)=-\left(v_{4}-v_{3}\right), \\
D_{p}\left(v_{4}-v_{3}\right)=-\left(\sigma_{4}-\sigma_{3}\right), \\
D_{p}\left(E_{4}-E_{3}\right)=-\left(\sigma_{4} v_{4}-\sigma_{3} v_{3}\right) .
\end{gathered}
$$

(4) Forward rarefaction wave:

$$
\begin{gathered}
s_{4}=s_{r}, \\
v_{4}=v_{r}-\int_{w_{r}}^{w_{4}}\left(\sigma_{w}\left(w, s_{r}\right)\right)^{1 / 2} d w .
\end{gathered}
$$

Since we have in total eleven unknowns and eleven equations including (2.1.4), solving this nonlinear system gives us the intermediate states $U_{1}, U_{3}$ and $U_{4}$. Our numerical tests show that the solution obtained by the nonlinear system is almost identical with our exact solution with suitable starting values of unknowns. In our numerical tests, we use the function fsolve() in Matlab ([20]) to solve the nonlinear system.

1.4.2. Exact Riemann Solver (II): Iterative Method. Although we can find the solution by solving a nonlinear system, it is a time-consuming job and the process may not converge if the starting values of the unknown variables are not good enough. In this part, we propose an iterative method which only require solving a "small" nonlinear system at each step. Here we show how to find the solution involving a forward phase boundary through this procedure. The case with a backward phase boundary is almost the same. We denote the projections of the forward phase boundary curve onto $\sigma v$-plane by $v=P_{3}(\sigma)$ (see Fig1.4.2). As we have cross the contact discontinuity that

$$
\begin{aligned}
& \sigma_{1}=\sigma_{3}, \\
& v_{1}=v_{3},
\end{aligned}
$$

the intersection point of $P_{1}(\sigma)$ and $P_{3}(\sigma)$ is the projection of the intermediate state $U_{3}$. However, it is expensive to use an iterative process to find this intersection 


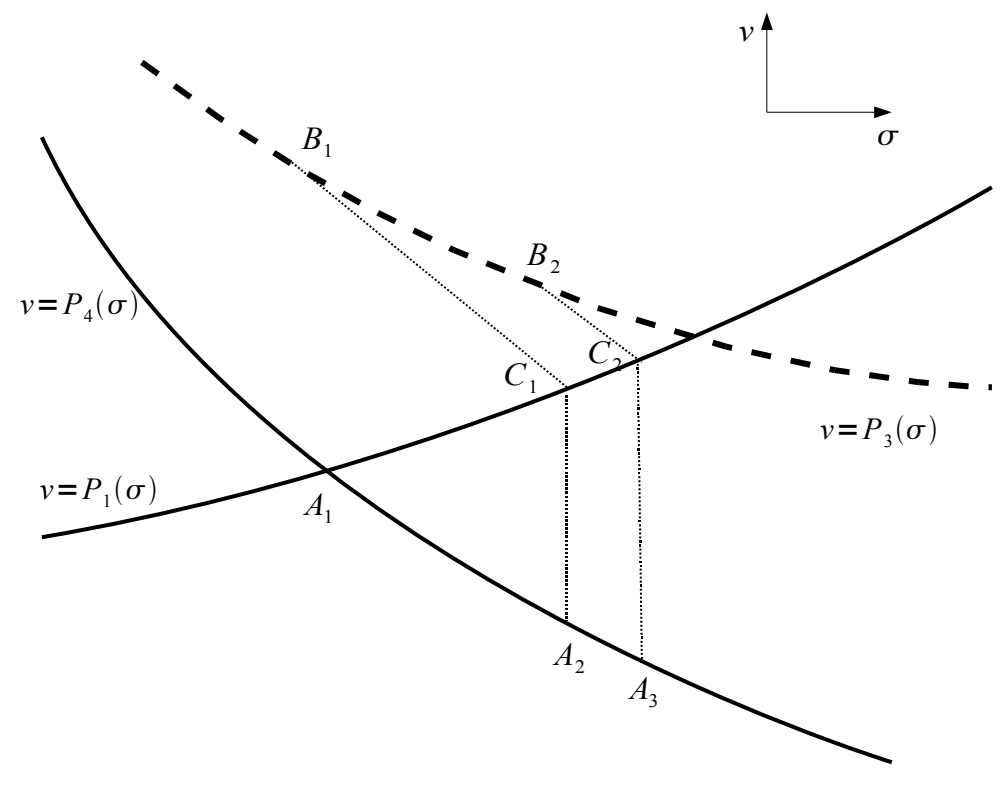

Figure 1.4.2. The Exact Riemann Solver II: Iterative Method.

point because for any given value $\sigma_{3}$, we need to solve a $6 \times 6$ or a $7 \times 7$ system to find $v_{3}$ (it depends on whether the 3 -wave is a rarefaction wave or a shock). So we suggest the following procedure in which we calculate the slope of $P_{4}(\sigma)$ instead of $P_{3}(\sigma)$ assuming that they do not differ significantly.

Step1 and step2 of the iterative process are exactly the same with the Exact Riemann Solver(I). We assume that condition (3) in Theorem 4.2 is satisfied so that the phase boundary moves forward. Denote by $A_{1}$ the intersection point of the $\sigma v$-projections of the shock-rarefaction curves connected to $U_{l}$ and $U_{r}$, respectively (Fig1.4.2).

Step3: Find the state $U_{4}$ whose projection onto $\sigma v$-plane is $A_{1}$ and find the state $U_{3}$ connected to $U_{4}$ through a forward phase boundary (Fig1.2.2). This step needs to solve a $4 \times 4$ system as follows.

$$
\begin{gathered}
D_{p}\left(w_{4}-w_{3}\right)=-\left(v_{4}-v_{3}\right), \\
D_{p}\left(v_{4}-v_{3}\right)=-\left(\sigma_{4}-\sigma_{3}\right), \\
D_{p}\left(E_{4}-E_{3}\right)=-\left(\sigma_{4} v_{4}-\sigma_{3} v_{3}\right), \\
D_{p}=\epsilon\left(s_{3}-s_{4}\right) .
\end{gathered}
$$

The projection of $U_{3}$, denoted by $B_{1}$, lies on the curve $P_{3}(\sigma)$ (Fig1.4.2).

Step4: Let $m_{1}$ be the slope of the tangent line to $v=P_{4}(\sigma)$ at $A_{1}$ (refer to section1.4 for details of calculation of $\left.m_{1}\right)$. Find the intersection point of the straight line passing through $B_{1}$ whose slope is $m_{1}$ with the curve $v=P_{1}(\sigma)$. This intersection point is denoted by $C_{1}$.

Step5: Find the point $A_{2}$ on $P_{4}(\sigma)$ that shares the same value of $\sigma$ with $C_{1}$. 


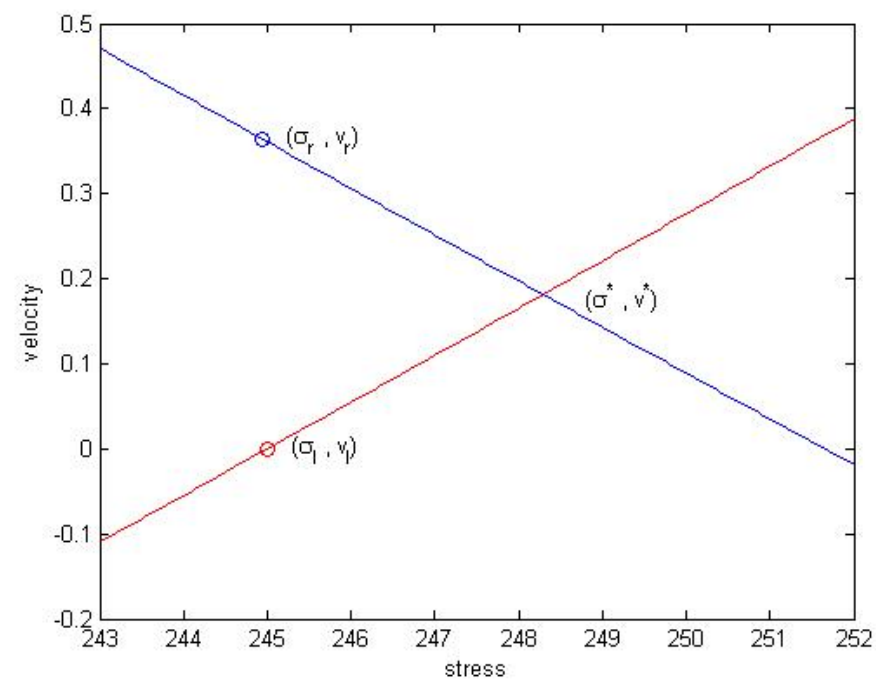

FigURE 1.5.1. $\sigma v$-projections of the shock-rarefaction curves.

Go back to step3 to find $B_{2}$ and repeat the process.

\subsection{Numerical Tests}

In numerical tests, we assume that the left state of the Riemann problem is specified in low-strain state (or $\alpha$-phase) and right state in high-strain state (or $\beta$-phase). We choose $\epsilon=0.1$ in the kinetic relation and the following constants (Table1) in the model of [21] .

TABle 1. Constants.

\begin{tabular}{ccccccc}
\hline$A$ & $B$ & $K$ & $C$ & $T_{0}$ & $W_{a}$ & $W_{b}$ \\
\hline 1 & 1 & 100 & 500 & 300 & 1 & 3 \\
\hline
\end{tabular}

1.5.1. Stationary Phase Boundary. As the stationary phase boundary coincides with the contact discontinuity in $x t$-plane, the solution of the Riemann problem has the configuration shown in Fig1.2.1 where the 1 or 3 -wave might be a shock or a rarefaction wave.

1.5.1.1. Test1. The initial values of the Riemann problem are given in Table2

TABLE 2. Initial value of Riemann problem.

\begin{tabular}{cccc}
\hline & $w$ & $v$ & $\sigma$ \\
\hline$U_{l}$ (low-strain state) & 0.8 & 0 & 245 \\
\hline$U_{r}$ (high-strain state) & 3.2 & 0.36468459 & 244.93011330 \\
\hline
\end{tabular}

The projections of the shock-rarefaction curves connected to $U_{l}$ and $U_{r}$ are depicted in Fig1.5.1 where the backward wave is a rarefaction wave and forward wave is a shock. The entropies are shown in Table3. 


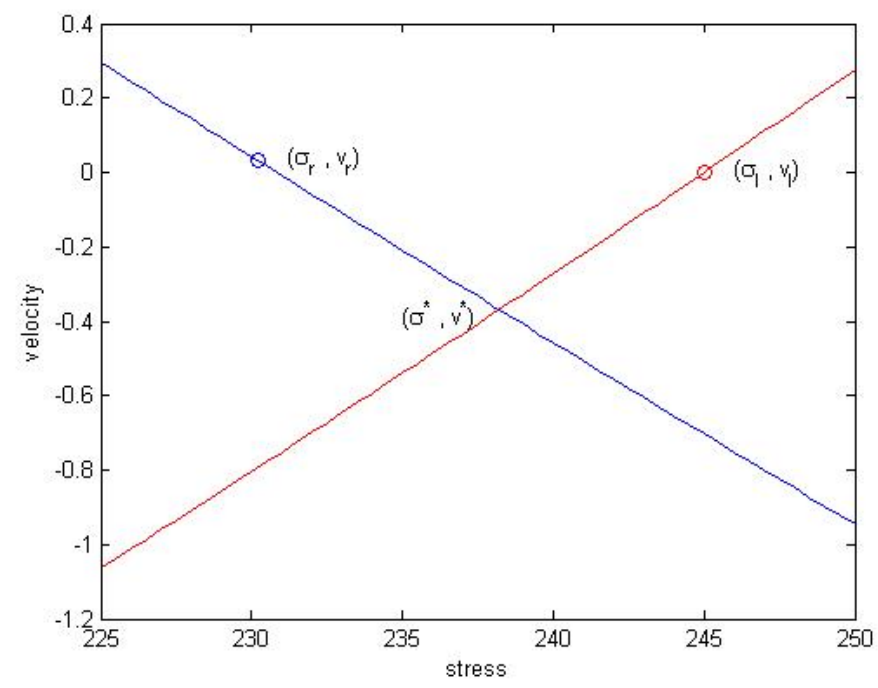

FiguRE 1.5.2. $\sigma v$-projections of the shock-rarefaction curves.

TABLE 3. The entropies.

\begin{tabular}{ccc}
\hline$s^{m}$ & $s_{1}$ & $s_{4}$ \\
\hline 401.39 & 493.84 & 271.39 \\
\hline
\end{tabular}

As $s_{1} \geq s^{m}$ and $s_{4} \leq s^{m}$, by Theorem $4.2(1)$, the phase boundary is stationary and the calculation is finished. Solution is shown in Table4.

TABLE 4. Solution with a stationary phase boundary (SPB).

\begin{tabular}{cccccc}
\hline & $w_{1}$ & $v_{1}$ & $\sigma_{1}\left(\times 10^{2}\right)$ & $w_{4}$ & $D_{3}$ \\
\hline Exact Solution & 0.81000000 & 0.18138367 & 2.48290036 & 3.2100000 & 18.3300921 \\
\hline Numerical Solution & 0.81000028 & 0.18138879 & 2.48290036 & 3.2100003 & 18.3295904 \\
\hline
\end{tabular}

where we omit $\sigma_{4}$ and $v_{4}$ as $\sigma_{4}=\sigma_{1}$ and $v_{4}=v_{1}$.

1.5.1.2. Test2. The initial values of the Riemann problem are given in Table5

TABLE 5. Initial value of Riemann problem.

\begin{tabular}{cccc}
\hline & $w$ & $v$ & $\sigma$ \\
\hline$U_{l}$ (low-strain state) & 0.8 & 0 & 245 \\
\hline$U_{r}$ (high-strain state) & 3.28 & 0.03107597 & 230.21262816 \\
\hline
\end{tabular}

The projections of the shock-rarefaction curves connected to $U_{l}$ and $U_{r}$ are depicted in Fig1.5.2 where both the backward and forward wave are shocks. The entropies are shown in Table6. 
TABle 6. The entropies.

\begin{tabular}{ccc}
\hline$s^{m}$ & $s_{1}$ & $s_{4}$ \\
\hline 380.57 & 493.84 & 141.73 \\
\hline
\end{tabular}

As $s_{1} \geq s^{m}$ and $s_{4} \leq s^{m}$, by Theorem 4.2 (1), the phase boundary is stationary and the calculation is finished. The solution is in Table7

TABLE 7. Solution with a stationary phase boundary (SPB).

\begin{tabular}{rcrrc}
\hline & $w_{1}$ & $v_{1}$ & $\sigma_{1}\left(\times 10^{2}\right)$ & $w_{4}$ \\
\hline Exact Solution & 0.78 & -0.36869149 & 2.38203329 & 3.3 \\
\hline Numerical Solution & 0.78000022 & -0.36868733 & 2.38203329 & 3.3000002 \\
\hline & & $D_{1}$ & $D_{3}$ \\
\\
\cline { 2 - 4 } & Exact Solution & -18.4345745 & 19.9883729 & \\
\cline { 2 - 3 } & Numerical Solution & -18.4347827 & 19.9881808 \\
& &
\end{tabular}

where we omit $\sigma_{4}$ and $v_{4}$ as $\sigma_{4}=\sigma_{1}$ and $v_{4}=v_{1}$.

\subsubsection{Non-stationary Phase Boundary.}

1.5.2.1. Test1. The initial values of the Riemann problem are given in Table8

TABLE 8. Initial value of Riemann problem.

\begin{tabular}{cccc}
\hline & $w$ & $v$ & $\sigma\left(\times 10^{2}\right)$ \\
\hline$U_{l}$ (low-strain state) & 0.98 & -1.0 & 2.30 \\
\hline$U_{r}$ (high-strain state) & 3.24 & 1.91406613 & 2.59370560 \\
\hline
\end{tabular}

Assuming that the phase boundary is stationary and we can find the solution (see Fig1.5.3 upper)

TABLE 9. Solution by assuming that the phase boundary is stationary.

\begin{tabular}{ccccc}
\hline$w_{1}$ & $v_{1}$ & $\sigma_{1}\left(\times 10^{2}\right)$ & $w_{4}$ & $D_{3}$ \\
\hline 1.20320351 & 1.70768829 & 2.63317144 & 3.25079219 & 19.1229752 \\
\hline
\end{tabular}

and we can determine that the 1 -wave is a rarefaction wave and the 3 -wave is a shock. Moreover, we obtain the entropies (Table10).

TABLE 10. The entropies.

\begin{tabular}{ccc}
\hline$s^{m}$ & $s_{1}$ & $s_{4}$ \\
\hline 430.89 & 372.91 & 272.86 \\
\hline
\end{tabular}

As $s_{1}<s^{m}$ and $s_{4} \leq s^{m}$, the solution contains a backward phase boundary by Theorem 4.2 (2) (Fig1.2.3) . Therefore the solution can be found by solving 
the nonlinear system that contains all the relations including the kinetic relation (Exact Riemann Solver I).

In addition, the $\sigma v$-projections of the shock-rarefaction curves as well as the backward phase boundary curves are depicted in Fig1.5.3(lower). The intersection of $v=P_{4}(\sigma)$ and $v=P_{1}(\sigma)$ is the projection of the solution with an SPB just obtained and will be used as starting point in our Exact Riemann Solver II. The intersection of $v=P_{4}(\sigma)$ and $v=P_{2}(\sigma)$ (dashed curve) is the projection of $U_{2}$ (or $U_{4}$ ) of the exact solution involving a BPB.

Here is the numerical results using the Exact Riemann Solver I and Exact Riemann Solver II for one iteration (Table11).

TABLE 11. Solution with a backward phase boundary.

\begin{tabular}{cccccc}
\hline & $w_{1}$ & $v_{1}$ & $\sigma_{1}\left(\times 10^{2}\right)$ & $w_{2}$ & $v_{2}$ \\
\hline Exact Solution & 1.2 & 1.67701179 & 2.63023396 & 3.11745912 & 1.72291346 \\
\hline Riemann Solver I & 1.200000 & 1.67701179 & 2.63023396 & 3.11745912 & 1.72291346 \\
\hline Riemann Solver II & 1.199992 & 1.67693725 & 2.63022679 & 3.11745652 & 1.72283792 \\
\hline \multicolumn{7}{c}{$\sigma_{2}\left(\times 10^{2}\right)$} & $w_{4}$ & $D_{3}$ & $D_{p}\left(\times 10^{-2}\right)$ \\
\hline Exact Solution & 2.63024495 & 3.25 & 19.1152670 & -2.393880 \\
\hline Riemann Solver I & 2.63024495 & 3.250000 & 19.1152670 & -2.393880 \\
\hline Riemann Solver II & 2.63023778 & 3.249998 & 19.1152480 & -2.393821 \\
\hline
\end{tabular}

where we skip $\sigma_{4}$ and $v_{4}$ since $\sigma_{2}=\sigma_{4}$ and $v_{2}=v_{4}$.

1.5.2.2. Test2. The initial values of the Riemann problem are given by

TABLE 12. Initial value of Riemann problem.

\begin{tabular}{cccc}
\hline & $w$ & $v$ & $\sigma\left(\times 10^{2}\right)$ \\
\hline$U_{l}$ (low-strain state) & 0.8 & 0 & 2.45 \\
\hline$U_{r}$ (high-strain state) & 3.01 & -2.82112850 & 2.73195121 \\
\hline
\end{tabular}

Assuming that the phase boundary is stationary and we can find the solution (see Fig1.5.4 upper)

TABLE 13. Solution by assuming that the phase boundary is stationary.

\begin{tabular}{ccccc}
\hline$w_{1}$ & $v_{1}$ & $\sigma_{1}\left(\times 10^{2}\right)$ & $w_{4}$ & $D_{1}$ \\
\hline 0.789257584 & -0.197050114 & 2.41385478 & 2.79040287 & -18.3431594 \\
\hline
\end{tabular}

and we can determine that the 1 -wave is a rarefaction wave and the 3 -wave is a shock. Moreover, we obtain the entropies (Table14).

TABle 14. The entropies.

\begin{tabular}{ccc}
\hline$s^{m}$ & $s_{1}$ & $s_{4}$ \\
\hline 387.23 & 493.84 & 444.62 \\
\hline
\end{tabular}



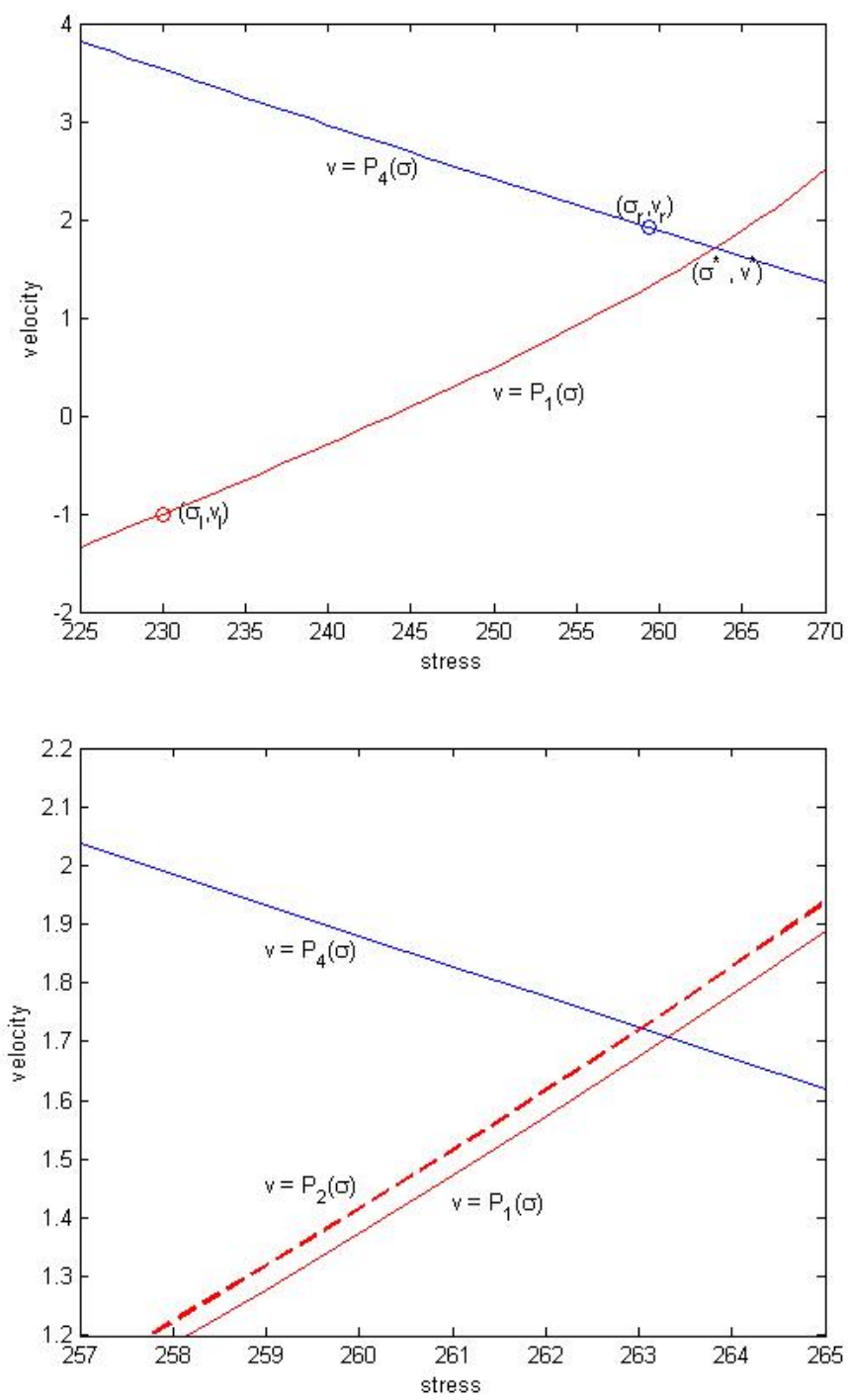

Figure 1.5.3. (Upper)The intersection point $\left(\sigma^{*}, v^{*}\right)$ of the $\sigma v$-projections of the shock-rarefaction curves. (Lower)The $\sigma v$ projections of the shock-rarefaction curves and the backward phase boundary curves.

As $s_{1}>s^{m}$ and $s_{4}>s^{m}$, the solution contains a forward phase boundary by Theorem 4.2 (3) (Fig1.2.2). Therefore the solution can be found by solving the nonlinear system that contains all the relations including the kinetic relation (Exact Riemann Solver I).

In addition, the $\sigma v$-projections of the shock-rarefaction curves as well as the backward phase boundary curves are depicted in Fig1.5.4 (lower). The intersection of $v=P_{4}(\sigma)$ and $v=P_{1}(\sigma)$ is the projection of the solution with an SPB just 

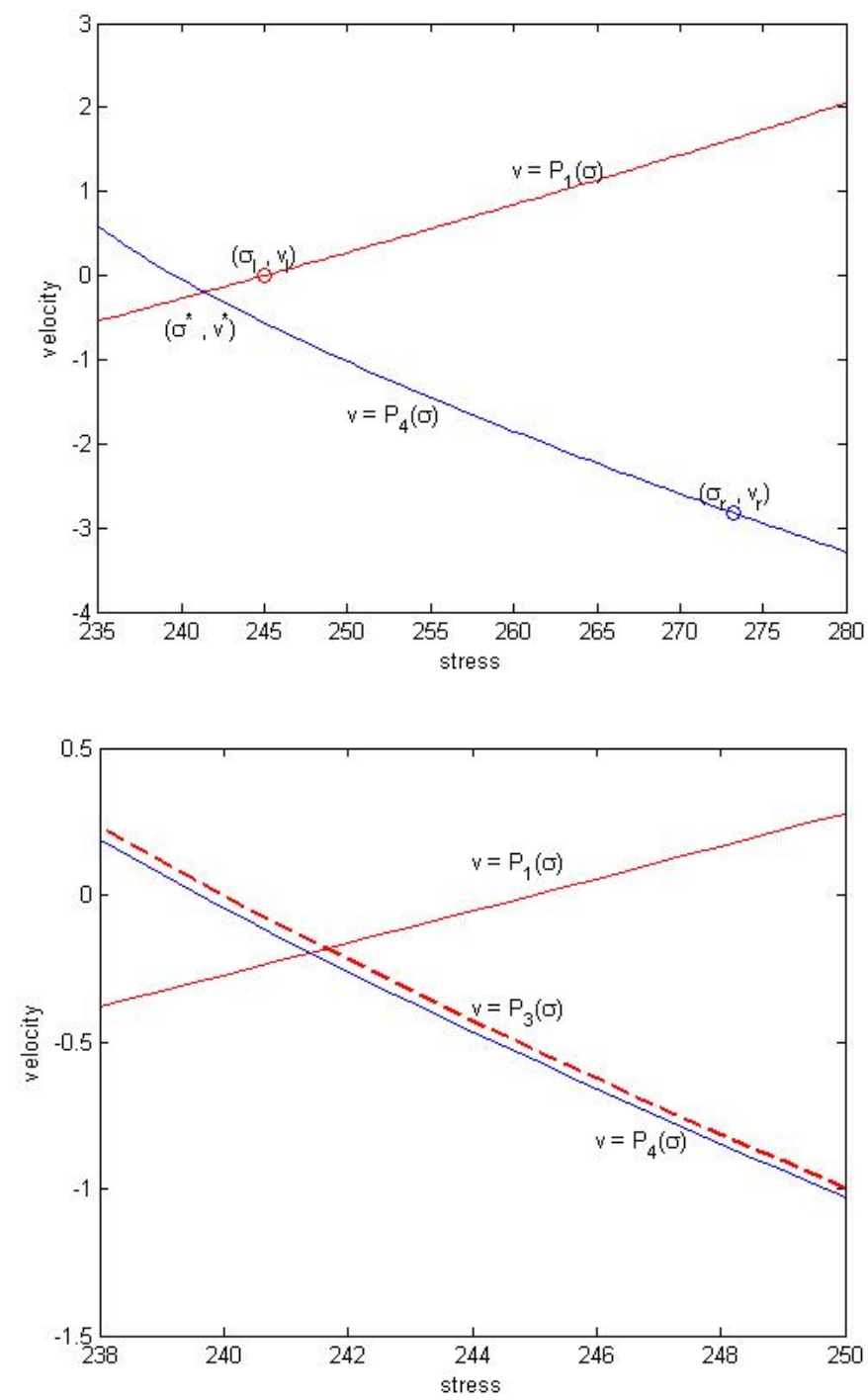

Figure 1.5.4. (Upper)The intersection point $\left(\sigma^{*}, v^{*}\right)$ of the $\sigma v$-projections of the shock-rarefaction curves. (Lower)The $\sigma v$ projections of the shock-rarefaction curves and the forward phase boundary curves.

obtained and will be used as starting point in our Exact Riemann Solver II. The intersection of $v=P_{3}(\sigma)$ and $v=P_{1}(\sigma)$ (dashed curve) is the projection of $U_{3}$ (or $U_{1}$ ) of the exact solution involving a FPB.

Here is the numerical results using the Exact Riemann Solver I and the Exact Riemann Solver II for one iteration (Table15). 
TABLE 15. Solution with a forward phase boundary.

\begin{tabular}{cccccc}
\hline & $w_{1}$ & $v_{1}$ & $\sigma_{1}\left(\times 10^{2}\right)$ & $w_{3}$ & $w_{4}$ \\
\hline Exact Solution & 0.79 & -0.18335855 & 2.41637964 & 0.88 & 2.7932611 \\
\hline Riemann Solver I & 0.7900000 & -0.18335855 & 2.41637964 & 0.8800000 & 2.7932611 \\
\hline Riemann Solver II & 0.7900036 & -0.18329196 & 2.41639192 & 0.8800013 & 2.7932652 \\
\hline \hline \multicolumn{2}{c}{$v_{4}$} & $\sigma_{4}\left(\times 10^{2}\right)$ & $D_{1}$ & $D_{p}\left(\times 10^{-2}\right)$ \\
\hline Exact Solution & -0.22395908 & 2.41638826 & -18.3358551 & 2.122059 \\
\hline Riemann Solver I & -0.22395909 & 2.41638826 & -18.3358551 & 2.122059 \\
\hline Riemann Solver II & -0.22399780 & 2.41639192 & -18.3358194 & 2.122033 \\
\hline
\end{tabular}

where we skip $\sigma_{3}$ and $v_{3}$ as $\sigma_{3}=\sigma_{1}$ and $v_{3}=v_{1}$.

1.5.2.3. Test3. The initial values of the Riemann problem are given by

TABLE 16. Initial value of Riemann problem.

\begin{tabular}{cccc}
\hline & $w$ & $v$ & $\sigma\left(\times 10^{2}\right)$ \\
\hline$U_{l}$ (low-strain state) & 0.8 & -0.1 & 2.45 \\
\hline$U_{r}$ (high-strain state) & 3.01 & -1.31651455 & 2.91996091 \\
\hline
\end{tabular}

Assuming that the phase boundary is stationary and we can find the solution (see Fig1.5.5 upper)

TABLE 17. Solution by assuming that the phase boundary is stationary.

\begin{tabular}{cccc}
\hline$w_{1}$ & $v_{1}$ & $\sigma_{1}\left(\times 10^{2}\right)$ & $w_{4}$ \\
\hline 0.849299215 & 0.77499959 & 2.65534186 & 2.84349965 \\
\hline
\end{tabular}

and we can determine that both of the 1-wave and the 3 -wave are rarefaction waves. Moreover, we obtain the entropies (Table18).

TABLE 18. The entropies.

\begin{tabular}{ccc}
\hline$s^{m}$ & $s_{1}$ & $s_{4}$ \\
\hline 435.09 & 502.19 & 478.25 \\
\hline
\end{tabular}

As $s_{1}>s^{m}$ and $s_{4}>s^{m}$, the solution contains a forward phase boundary by Theorem 4.2 (3) (Fig1.2.2). Therefore the solution can be found by solving the nonlinear system that contains all the relations including the kinetic relation (Exact Riemann Solver I).

In addition, the $\sigma v$-projections of the shock-rarefaction curves as well as the backward phase boundary curves are depicted in Fig1.5.5 (lower). The intersection of $v=P_{4}(\sigma)$ and $v=P_{1}(\sigma)$ is the projection of the solution with an SPB just obtained and will be used as starting point in our Exact Riemann Solver II. The intersection of $v=P_{3}(\sigma)$ and $v=P_{1}(\sigma)$ (dashed curve) is the projection of $U_{3}$ (or $\left.U_{1}\right)$ of the exact solution involving a FPB. 

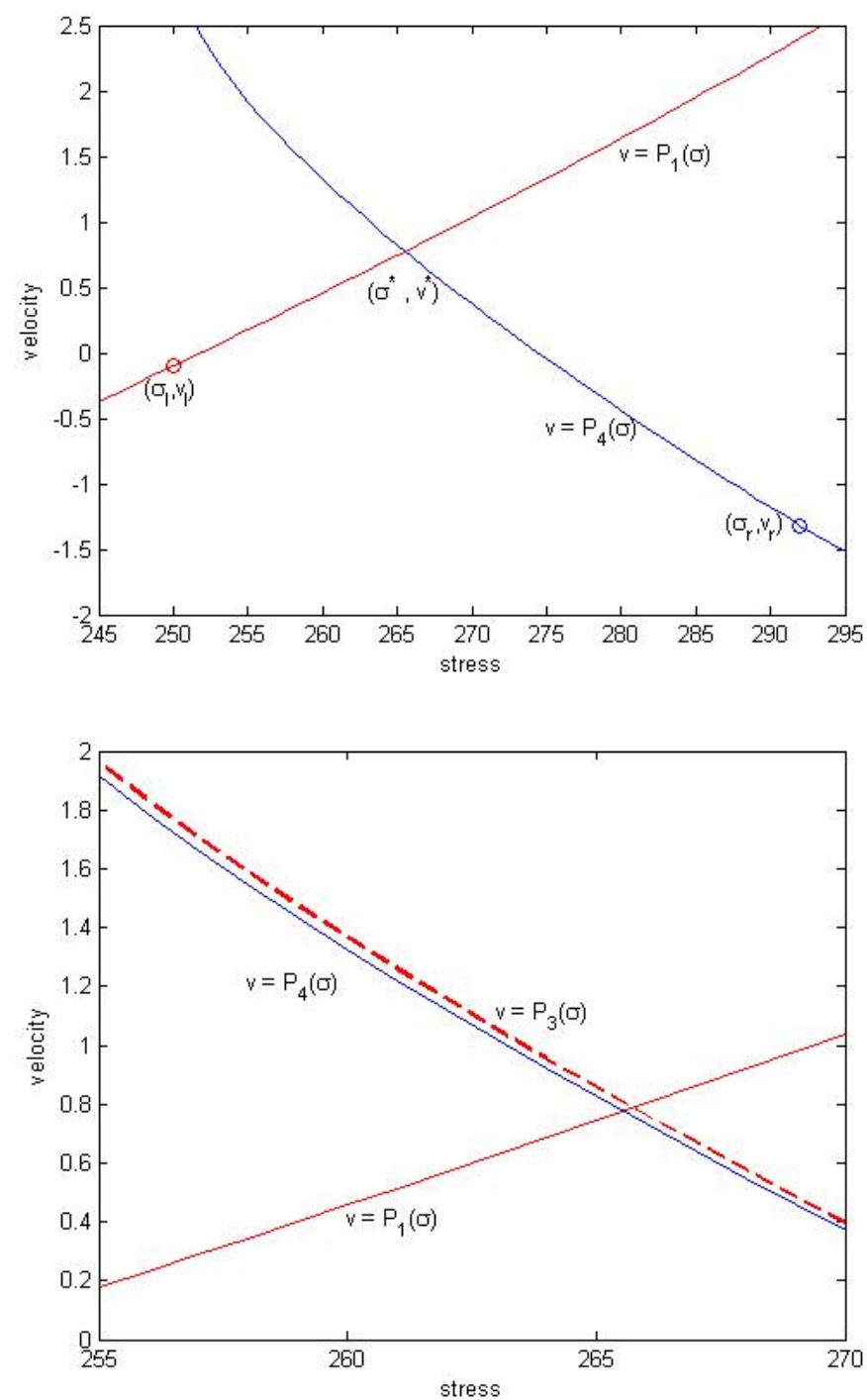

Figure 1.5.5. (Upper)The intersection point $\left(\sigma^{*}, v^{*}\right)$ of the $\sigma v$-projections of the shock-rarefaction curves. (Lower)The $\sigma v$ projections of the shock-rarefaction curves and the forward phase boundary curves.

Here is the numerical results using the Exact Riemann Solver I and the Exact Riemann Solver II for one iteration (Table19). 
TABle 19. Solution with a forward phase boundary.

\begin{tabular}{cccccc}
\hline & $w_{1}$ & $v_{1}$ & $\sigma_{1}\left(\times 10^{2}\right)$ & $w_{3}$ & $w_{4}$ \\
\hline Exact Solution & 0.85 & 0.78708989 & 2.65742682 & 0.9 & 2.8453259 \\
\hline Riemann Solver I & 0.850000 & 0.78708990 & 2.65742682 & 0.89999999 & 2.8453259 \\
\hline Riemann Solver II & 0.850003 & 0.78714163 & 2.65743574 & 0.90000340 & 2.8453337 \\
\hline & & $v_{4}$ & $\sigma_{4}\left(\times 10^{2}\right)$ & $D_{p}\left(\times 10^{-2}\right)$ \\
\hline Exact Solution & 0.755470088 & 2.65743195 & 1.62542422 \\
\cline { 2 - 5 } \\
\cline { 2 - 5 } Riemann Solver I & 0.755470107 & 2.65743196 & 1.62542418 \\
\hline
\end{tabular}

where we skip $\sigma_{3}$ and $v_{3}$ as $\sigma_{3}=\sigma_{1}$ and $v_{3}=v_{1}$. 


\section{CHAPTER 2}

\section{$L^{1}$ Well Posedness of Euler Equations With Dynamic Phase Boundaries}

\subsection{Introduction}

We study the well posedness of the Cauchy problem to Euler equations with two phase boundaries moving in opposite directions. The system is given by

$$
\begin{aligned}
v_{t}-u_{x} & =0, \\
u_{t}-f_{x} & =0, \\
E_{t}-(u f)_{x} & =0,
\end{aligned}
$$

where $v, u$ and $E$ are strain, velocity and total energy respectively. $f=-p$ is stress where $p$ is pressure. The total energy $E$ is given by $E=e+\frac{1}{2} u^{2}$, where $e$ is the internal energy and $u^{2} / 2$ is the kinetic energy. We take strain and entropy $s$ as state variables. Therefore, $e$ and $f$ are functions of $v$ and $s$. The solution to the system is written as $U=(v, u, s)$. We assume that $e$ is a smooth function of $v$ and $s, e_{s}=T>0$, and $e_{v s}=T_{v}<0$, where $T$ is temperature. We have the following thermodynamic relation

$$
d e=f d v+T d s .
$$

The initial value of (2.1.1) is given by

$$
U(0, x)=\bar{U}=(\bar{v}, \bar{u}, \bar{s})= \begin{cases}\bar{U}_{1} & x<x_{1}, \\ \bar{U}_{2} & x_{1}<x<x_{2}, \\ \bar{U}_{3} & x>x_{2},\end{cases}
$$

where $\bar{U}_{i}(i=1,2,3)$ are perturbed constant states. Specifically, there exists a function $U^{c}$ that takes constant value on each of the intervals $\left(-\infty, x_{1}\right),\left(x_{1}, x_{2}\right)$ and $\left(x_{2},+\infty\right)$ satisfying $\bar{U}-U^{c} \in L^{1} \cap B V$ with total variation sufficiently small. We assume that the solution contains two phase boundaries, denoted by $P_{1}$ and $P_{2}$ respectively, moving in opposite directions (see Fig2.1.1). We consider one interesting case in physics [11] where

the speed of a phase boundary is close to zero such that a phase boundary moves much slower than any shock (except for contact discontinuity) or rarefaction wave.

We also assume that there exists a constant $c_{0}$ such that every level curve $f(v, s)=c$, where $c$ is a constant satisfying $c>c_{0}$, is non-monotone in the $v s$-plane (see Fig2.1.2 where the curve $f_{v}=0$ is also sketched). Note that $f_{v}<0$ is inside the curve $f_{v}=0$. This region where $f_{v}<0$ is called the spinodal region and the states with the values of $(v, s)$ in this region are physically unstable and are not 


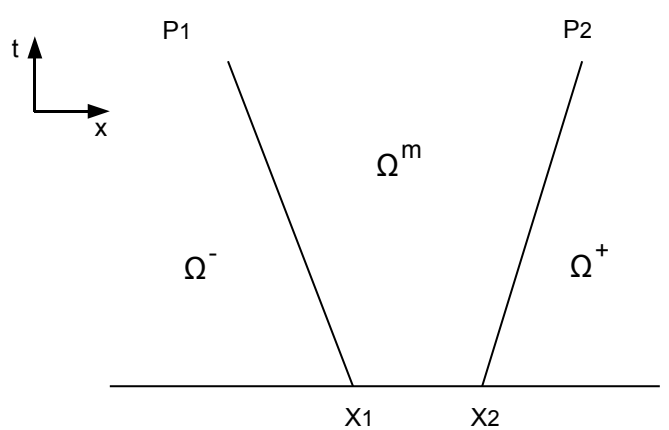

Figure 2.1.1. The solution to the initial value problem (2.1.1) (2.1.3). The upper $x t$-plane is divided into three regions $\Omega^{-}, \Omega^{m}$ and $\Omega^{+}$by the phase boundaries $P_{1}$ and $P_{2}$.

observable. For $s<s_{c}$, the region where $f_{v}>0$ is separated into two sub-regions. If $v$ is on the left and right of $f_{v}=0$ and $f_{v}(v, s)>0, v$ is said to be in the $\alpha$-phase and $\beta$-phase, respectively. In the region where $s<s_{c}$ and $f_{v}>0$, we assume that $f_{v v} \neq 0$ so that the system is genuinely nonlinear. A typical material satisfying the above assumptions at least locally in the $\alpha$ and $\beta$-phase is the van der Waals fluid where

$$
f=-\frac{8 e^{3 s /(8 a)}}{(3 v-1)^{1+1 / a}}+\frac{3}{v^{2}}
$$

with the positive constant $a$.

As the weak solutions to the initial value problem with phase transition are not unique, we use the entropy condition and the kinetic relation as the admissibility criteria to select a physically relevant solution. The entropy condition imposes that the entropy increases across jump discontinuities. The rate of decay of the entropy is given by

$$
E\left(v_{-}, s_{-}, v_{+}, s_{+}\right)=\sigma\left(v_{-}, v_{+}\right)\left(s_{+}-s_{-}\right),
$$

where $\sigma\left(v_{-}, v_{+}\right)= \pm \sqrt{\frac{f_{+}-f_{-}}{v_{+}-v_{-}}}$is the speed of the jump discontinuity and the subscripts - and + denote the states to the left and right of the discontinuity, respectively. The entropy condition requires that $E\left(v_{-}, s_{-}, v_{+}, s_{+}\right) \leq 0$ holds across each discontinuity.

The kinetic relation is proposed by Abeyaratne and Knowles $[\mathbf{1}, \mathbf{3}]$. It postulates that there exists a non-decreasing function $\phi(g)$ satisfying $\phi(0)=0$, where $g$ is called the driving traction, such that the speed of discontinuity is given by

$$
\sigma=\phi(g) .
$$

In order that this relation is consistent with the entropy condition, we require that $\phi^{\prime}>0$ so that $\sigma g>0$ holds. In this paper we choose $g=-\left(s_{+}-s_{-}\right)$for the driving 


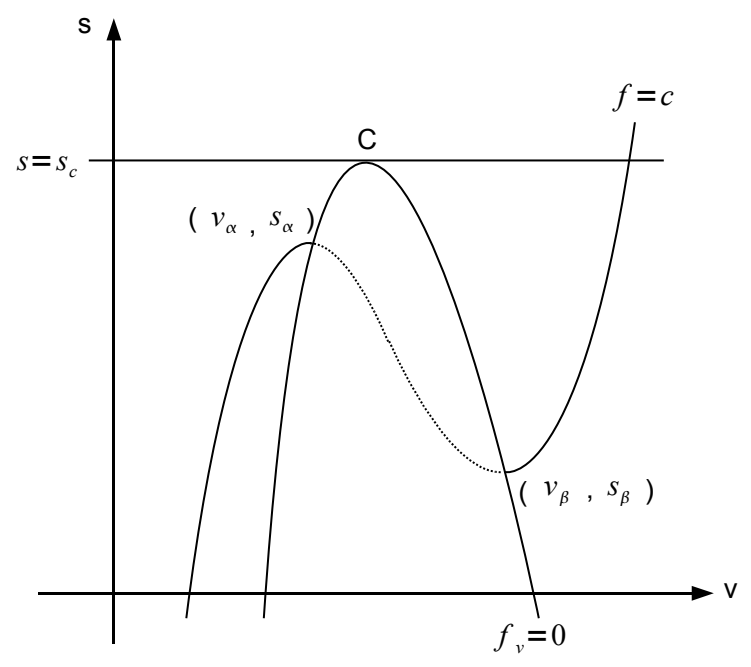

Figure 2.1.2. An example of a level curve $f(v, s)=c$.

traction. In particular we use the following kinetic condition

$$
\sigma_{p}=\epsilon\left(s_{-}-s_{+}\right),
$$

where $\epsilon$ is a small positive constant. This relation is applied to the solutions satisfying the entropy condition.

We also use the initiation criterion which has been used in $[\mathbf{1}, \mathbf{3}, \mathbf{1 3}]$.This criterion imposes that no new phase boundary occurs from any point unless no solution exists without the creation of a new phase. This ensures that the spontaneous initiation of a new phase cannot occur from two nearby initial states in the same phase.

In the absence of phase change, J. Glimm proved the existence of the weak solution in the space of bounded variations in his classical paper [10] for $n \times n$ hyperbolic system when the initial data are sufficiently small in $B V$. Bressan, Crasta and Piccoli $[7]$ proved that this problem is well posed when the total variation of the initial data $u_{0} \in L^{1} \cap B V$ is sufficiently small and they showed that the entropy solutions constitute a semigroup which is Lipschitz continuous with respect to time and initial data. Their analysis of stability was then simplified by Bressan, Liu and Yang in [8] where they introduced a functional that is equivalent to the $L^{1}$ distance between two different solutions and they showed that this functional is almost decreasing with respective to time. In the case of $n \times n$ hyperbolic system with large initial data, Lewicka and Trivisa [17] considered the solution which contains two large shocks. They showed the existence of the weak solutions under suitable Finiteness Condition and the stability under the Stability Condition. In addition, Lewicka [15]considered a general case with $m$ large shocks, $2<m \leq n$, 
and showed existence and $L^{1}$ stability of the problem under similar Finiteness and Stability Conditions.

In the case of conservation laws involving phase transition, Hattori [12] discussed the existence of weak solutions with moving phase boundaries. He considered the case where there are two noninteracting phase boundaries moving in the opposite directions and obtained the existence in $B V$ provided that the wave speeds do not differ significantly between different phases. The case where the two phase boundaries collide was also mentioned.

The goal of this paper is to show the existence and $L^{1}$ stability of initial value problem (2.1.1) (2.1.3). As in $[\mathbf{8}, \mathbf{1 7}]$ we introduce a functional which is equivalent to the $L^{1}$ distance between two different solutions. We formulate a Finiteness Condition and a Stability Condition that are similar to those in [17]. We show that the Finiteness Condition guarantees the existence of the weak solution and the Stability Condition implies the stability and yields the existence of a Lipschitz semigroup of entropy solutions.

Hattori [12] obtained the existence of the solution in $B V$ to the initial value problem (2.1.1) (2.1.3) given that the wave speeds do not differ significantly between different phases. We show that both the Finiteness Condition and Stability Condition hold in this case, such that the weak solution not only exists but also is stable.

This paper consists of five sections. Section 2 is the preliminary where we summarize the solutions of the Riemann problems discussed in [12] and introduce the Finiteness Condition and Stability Condition. In Section 3, we introduce the front tracking approximation of the initial value problem and state the main theorem on existence. The $L^{1}$ Lyapunov functional is stated in Section 4 whose derivative with respective to time will be analyzed in Section 5 .

\subsection{Preliminaries}

In this section, we firstly summarize the results in [11] concerning the Riemann problems with dynamic phase transitions. The configuration of Riemann problems is essential in the front tracking approximations when a phase boundary collides with a small physical wave. Then we introduce the Finiteness Condition and Stability Condition that will play an important role in derivation of the existence and stability, respectively, of the initial value problem (2.1.1) (2.1.3).

2.2.1. Phase Boundaries. A phase boundary is a line of discontinuity in the $x t$-plane across which the phase changes. Similar to a shock, the phase boundary satisfies the Rankine-Hugoniot condition

$$
\begin{aligned}
\sigma_{P}\left(v-v_{0}\right) & =-\left(u-u_{0}\right), \\
\sigma_{P}\left(u-u_{0}\right) & =-\left(f-f_{0}\right), \\
\sigma_{P}\left(E-E_{0}\right) & =-\left(f u-f_{0} u_{0}\right),
\end{aligned}
$$

where $\sigma_{P}$ is the speed of discontinuity. However, a phase boundary does not belong to any characteristic family. The phase boundary curve $P\left(U_{0}\right)$ is the set of all possible states $U=(v, u, s)$ connected to $U_{0}=\left(v_{0}, u_{0}, s_{0}\right)$ by a phase boundary. The projection of $P\left(U_{0}\right)$ on to the $v s$-plane is called the Hugoniot locus and denoted by $H\left(v_{0}, s_{0}\right)$. As $v_{0}$ and $v$ are in the different phases, $H\left(v_{0}, s_{0}\right)$ is a semi-infinite curve in $v s$-plane with an end $\left(v_{0}^{*}, s_{0}^{*}\right)$ in the phase other than the one that $\left(v_{0}, s_{0}\right)$ 
lies in. In the following lemmas, we discuss the location of $\left(v_{0}^{*}, s_{0}^{*}\right)$ given $\left(v_{0}, s_{0}\right)$ and the relations between $s_{0}$ and $s_{0}^{*}$. This end point plays an important role in the Riemann problems. In what follows, we assume that $\left(v_{0}, s_{0}\right)$ and $\left(v_{0}^{*}, s_{0}^{*}\right)$ are in the $\alpha$-phase and $\beta$-phase, respectively. Integrating both sides of (2.1.2) along a level curve $C: f=f_{0}$ in the $v s$-plane from $\left(v_{0}, s_{0}\right)$ to $\left(v_{0}^{*}, s_{0}^{*}\right)$ gives

$$
e-e_{0}=f_{0}+\int_{C} e_{s} d s
$$

Therefore, we have the following lemma [11].

Lemma 2.1. If $e\left(v_{\beta}, s_{\beta}\right)-e_{0} \leq f_{0}\left(v_{\beta}-v_{0}\right)$, where $\left(v_{\beta}, s_{\beta}\right)$ is the state in the vs-plane at which $f_{v}=0$ along a level curve $f=f_{0}$ (Fig2.1.2), then in the vs-plane the Hugoniot locus $H\left(v_{0}, s_{0}\right)$ of the phase boundary curve $P\left(U_{0}\right)$ is a semi-infinite curve starting from the point $\left(v_{0}^{*}, s_{0}^{*}\right)$, with $v_{0}^{*}$ in the other phase, satisfying

$$
f\left(\left(v_{0}^{*}, s_{0}^{*}\right)=f_{0}, e_{0}^{*}-e_{0}=f_{0}\left(v_{0}^{*}-v_{0}\right) .\right.
$$

Furthermore, $\int_{C} e_{s} d s=0$ holds, where the integral is the path integral along $f=f_{0}$ from $\left(v_{0}, s_{0}\right)$ to $\left(v_{0}^{*}, s_{0}^{*}\right)$.

Next lemma can be regarded as an extension of the Maxwell equal area rule from the isothermal case to non-isothermal case.

Lemma 2.2. In the vs-plane, if the level curve $f=f_{0}$ is not monotone, there exists a unique state $\left(v_{0}^{m}, s_{0}^{m}\right)$ on $f\left(v_{0}^{m}, s_{0}^{m}\right)=f_{0}$ with $v_{0}^{m}$ in the $\alpha$-phase such that $\left(v_{0}^{*}, s_{0}^{*}\right)=\left(v_{0}^{*}, s_{0}^{m}\right)$, i.e., the Hugoniot locus of the phase boundary curve $P\left(U_{0}\right)$ starts from the same value of entropy. This also implies that if $s_{0}>s_{0}^{m}, s_{0}<s_{0}^{*}$ and if $s_{0}<s_{0}^{m}, s_{0}>s_{0}^{*}$. The corresponding result holds if $v_{0}^{m}$ is given in the $\beta$-phase.

We call the states $(v, s)$ in the $\alpha$-phase (or $\beta$-phase) "stable" if it satisfies that $s \leq s_{0}^{m}$ (or $s \geq s_{0}^{m}$ ) and the states $(v, s)$ in the $\alpha$-phase (or $\beta$-phase) "metastable" if $s>s_{0}^{m}\left(\right.$ or $\left.s<s_{0}^{m}\right)$.

2.2.2. The Riemann Problems. The initial data of a Riemann problem is given by

$$
U(0, x)=(v, u, s)(0, x)= \begin{cases}U_{l}=\left(v, u_{l}, s_{l}\right) & x<0 \\ U_{r}=\left(v_{r}, u_{r}, s_{r}\right) & x>0 .\end{cases}
$$

We seek a self-similar solution consisting of constant states separated by the backward and forward wave, the phase boundaries, and contact discontinuity or the stationary phase boundary. The backward and forward waves are shocks and rarefaction waves. In the case where the speeds of phase boundaries are much smaller than those of the forward and backward waves, we have the configuration of the phase boundaries given the following theorem. For the discussion of the cases where $v_{l}$ and $v_{r}$ in the same phase, refer to [11].

Theorem 2.3. If $v_{l}$ and $v_{r}$ are specified in different phases, there are four different solution configurations near $\sigma_{p}=0$ depending on the values of $s_{1}$ and $s_{4}$ at $\sigma_{p}=0$. 


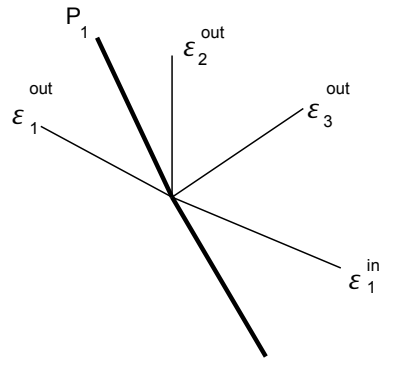

(a)

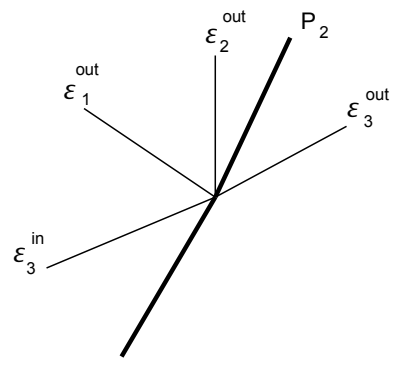

(b)

Figure 2.2.1. The definition of $m_{13}^{P_{1}}(\mathrm{a})$ and $m_{31}^{P_{2}}(\mathrm{~b})$.

(1) If $s_{1} \leq s_{1}^{m}$ and $s_{4} \geq s_{4}^{m}$ at $\sigma_{P}=0$, then the solution with the stationary phase boundary is the only solution satisfying the entropy condition.

(2) If $s_{1}>s_{1}^{m}$ and $s_{4} \geq s_{4}^{m}$ at $\sigma_{P}=0$, there is a one-parameter family of solutions with the backward phase boundary.

(3) If $s_{1} \leq s_{1}^{m}$ and $s_{4}<s_{4}^{m}$ at $\sigma_{P}=0$, there is a one-parameter family of solutions with the forward phase boundary.

(4) If $s_{1}>s_{1}^{m}$ and $s_{4}<s_{4}^{m}$ at $\sigma_{P}=0$, there are three solution configurations; there are two one-parameter families of solutions, one with the backward phase boundary and another with forward phase boundary. Also it is possible to construct the solutions with three phase boundaries where the left phase boundary moves backward, the middle one is stationary and the right one moves froward. In this case we have a two-parameter family of solutions.

Furthermore, except Case (4) there is a unique solution satisfying the kinetic relation (2.1.4) provided that $\epsilon$ is sufficiently small. The details of the proof are available in [11]. In what follows, we choose the initial data such that Cases (2) and (3) in Theorem 2.2 will occur.

2.2.3. The Finiteness Condition and Stability Condition. Consider the wave interaction pattern in Fig2.2.1(a) where a 1-family wave impinges a backward phase boundary, denoted by $P_{1}$, from the right. As in [17], we define the number

$$
m_{13}^{P_{1}}=\frac{\partial \varepsilon_{3}^{\text {out }}}{\partial \varepsilon_{1}^{\text {in }}} .
$$

Similarly, if a 3 -family wave interacts with a forward phase boundary, denoted by $P_{2}$, from the left (see Fig2.2.1 (b)), we define

$$
m_{31}^{P_{2}}=\frac{\partial \varepsilon_{1}^{o u t}}{\partial \varepsilon_{3}^{\text {in }}} .
$$

We introduce the following Finiteness Condition and Stability Condition that can be regarded as extensions of those in [17] where the large shocks are replaced by phase boundaries. 
Finiteness Condition There exist positive weights $w_{1}, w_{3}$ and a number $\theta \in$ $(0,1)$ such that

$$
\frac{w_{3}}{w_{1}} \cdot\left|m_{13}^{P_{1}}\right|<\theta
$$

and

$$
\frac{w_{1}}{w_{3}} \cdot\left|m_{31}^{P_{2}}\right|<\theta
$$

where $w_{2}$ is a small fixed constant.

Stability Condition There exist positive weights $\tilde{w}_{1}, \tilde{w}_{3}$ and a number $\Theta \in$ $(0,1)$ such that

$$
\frac{\tilde{w}_{3}}{\tilde{w}_{1}} \cdot\left|m_{13}^{P_{1}}\right| \cdot\left|\frac{\lambda_{3}\left(U_{0}^{m}\right)-\sigma^{P_{1}}}{\lambda_{1}\left(U_{0}^{m}\right)-\sigma^{P_{1}}}\right|<\Theta
$$

and

$$
\frac{\tilde{w}_{1}}{\tilde{w}_{3}} \cdot\left|m_{31}^{P_{2}}\right| \cdot\left|\frac{\lambda_{1}\left(U_{0}^{m}\right)-\sigma^{P_{2}}}{\lambda_{3}\left(U_{0}^{m}\right)-\sigma^{P_{2}}}\right|<\Theta
$$

where $\tilde{w}_{2}$ is a small fixed constant and $U_{0}^{m}$ is a constant state in $\Omega^{m}$ defined in (2.3.3).

We will show in Section 3 the existence of the weak solutions of (2.1.1) and (2.1.3) under the Finiteness Condition. As $\lambda_{1}\left(U_{0}^{m}\right)=-\sqrt{f_{v}\left(U_{0}^{m}\right)}, \lambda_{3}\left(U_{0}^{m}\right)=$ $\sqrt{f_{v}\left(U_{0}^{m}\right)}$ and $\sigma^{P_{1}}<0<\sigma^{P_{2}}$, we have

$$
\left|\frac{\lambda_{3}\left(U_{0}^{m}\right)-\sigma^{P_{1}}}{\lambda_{1}\left(U_{0}^{m}\right)-\sigma^{P_{1}}}\right|>1
$$

and

$$
\left|\frac{\lambda_{1}\left(U_{0}^{m}\right)-\sigma^{P_{2}}}{\lambda_{3}\left(U_{0}^{m}\right)-\sigma^{P_{2}}}\right|>1 \text {. }
$$

Therefore, the Stability Condition is stronger than the Finiteness Condition (for general $n \times n$ hyperbolic system, see [16]). The Stability Condition is essential in the proof of stability in Section 4 and Section 5.

\subsection{Front Tracking Approximations}

Given the Cauchy problem (2.1.1)(2.1.3), we employ the strategy of $[\mathbf{5}, \mathbf{6}, \mathbf{1 7}]$ to obtain the existence of its solution as follows:

(i) Approximate the initial data $\bar{U}$ by piecewise constant data $\bar{U}_{\varepsilon}$.

(ii) Construct an "approximate solution" $U_{\varepsilon}$ to $(2.1 .1)$ with $U_{\varepsilon}(0, \cdot)=\bar{U}_{\varepsilon}$. The approximating function $U_{\varepsilon}$ is piecewise constant with finitely many jumps occurring along straight discontinuity lines. For example, the rarefaction wave will be approximated by finitely many small discontinuities.

(iii) Show that for some parameter sequence $\varepsilon_{n} \rightarrow 0$, the sequence $U_{\varepsilon_{n}}$ has a limit in $L_{l o c}^{1}$, and that this limit is a solution to (2.1.1) and (2.1.3).

As a phase boundary moves much slower than a forward or backward wave, for convenience, we call a backward phase boundary $\left(P_{1}\right)$ a $1 \frac{1}{2}$-family wave and a forward phase boundary $\left(P_{2}\right)$ a $2 \frac{1}{2}$-family wave. Note that a $1 \frac{1}{2}$-family wave or 
a $2 \frac{1}{2}$-family wave is just a notation indicating a slow backward or forward phase boundary. Therefore, we have in total 6 families of waves:

1-family: backward shock or rarefaction wave,

$1 \frac{1}{2}$-family: backward phase boundary,

2-family: contact discontinuity,

$2 \frac{1}{2}$-family: forward phase boundary,

3 -family: forward shock or rarefaction wave,

4-family: non-physical wave $[\mathbf{6}, \mathbf{5}, \mathbf{1 7}]$.

We denote by $\lambda_{k}$ the characteristic speed of a $k$-family wave for $k=1,2,3$. $\lambda_{1 \frac{1}{2}}=\sigma_{P_{1}}$ and $\lambda_{2 \frac{1}{2}}=\sigma_{P_{2}}$ represent the speeds of the backward and forward phase boundaries, respectively. The speed of a non-physical wave is usually written as $\hat{\lambda}$. Then we have $\lambda_{1}<\lambda_{1 \frac{1}{2}}<\lambda_{2}=0<\lambda_{2 \frac{1}{2}}<\lambda_{3}<\hat{\lambda}$. In our problem, the strengths of all waves are very small except for the phase boundaries.

In the construction of the wave front tracking method, we assume that at most two waves interact with each other at any moment. We solve the Riemann problem $\left(U_{l}, U_{r}\right)$ when interaction occurs. One problem in constructing the front tracking approximation is to keep the number of wave fronts finite for all times $t>0$ (see [5]). Therefore, we choose a threshold number $\varepsilon_{T}$ and solve a Riemann problem by the Accurate Riemann Solver when the product of the strengths of the colliding waves is greater than $\varepsilon_{T}$. When the product is less than $\varepsilon_{T}$, a Riemann problem is solved by the so-called Simplified Riemann Solver where we let the incoming waves pass through each other, changing their speeds slightly, and collect the remaining waves into the non-physical wave. If both states $U_{l}$ and $U_{r}$ are in the same set $\Omega^{-}, \Omega^{m}$ or $\Omega^{+}$, the wave interaction occurs in the same phase and we solve the Riemann problem as [5]. If a small wave interacts with a phase boundary, we solve the problem as follows.

\section{(i) Accurate Riemann Solver}

This is a self-similar solution with the rarefaction wave replaced by a piecewise constant rarefaction fan [17]. In this paper, we choose the initial value such that Case (2) and Case (3) in Theorem 2.3 occur. As the incoming wave is very small, the configuration of the phase boundary in Theorem 2.3 will not change after interaction.

(ii) Approximate Riemann Solver

Case 1 A physical wave of family $k(k=2,3)$ impinges a backward phase boundary from the left. The Riemann problem is solved as follows (see Fig2.3.1, where the case of $k=3$ is shown):

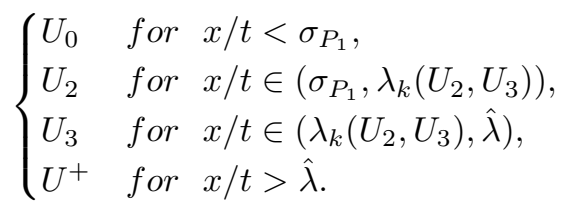




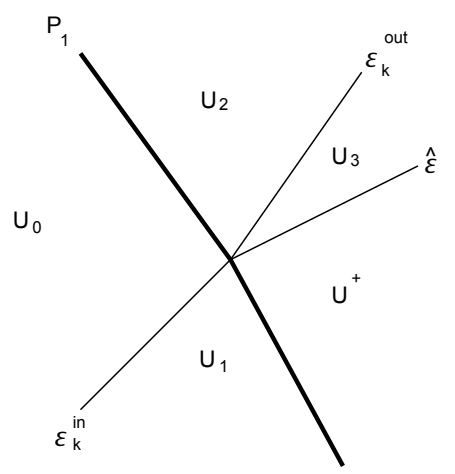

Figure 2.3.1. Approximate Riemann Solver.

where $U_{3}=\Psi_{k}\left(U_{2}, \varepsilon\right)$ is the state connected to $U_{2}$ through a 3 -family shockrarefaction curve (when $k=3$ ) or a contact discontinuity (when $k=2$ ). If $k=2$, $\lambda_{k}=0$. If $k=3$,

$$
\lambda_{3}\left(U_{2}, U_{3}\right)= \begin{cases}\lambda_{3}\left(U_{2}\right) & \text { if } \varepsilon>0 \\ \frac{u_{2}-u_{0}}{v_{0}-v_{2}} & \text { if } \varepsilon<0 .\end{cases}
$$

The middle state $U_{2}$ is defined as follows. Let $\Psi_{1 \frac{1}{2}}\left(U_{l}, U_{r}\right)=0$ be the backward phase boundary curve connecting the two states $U_{l}$ and $U_{r} . \Psi_{k}(k=1,3)$ is the $k$-family shock-rarefaction curve. In this case, we have $U_{1}=\Psi_{3}\left(U_{0}, \varepsilon^{i n}\right)$. We also use an equivalent expression $U_{0}=\tilde{\Psi}_{3}\left(U_{1},-\varepsilon^{i n}\right)$. $\Psi_{4}$ is the nonphysical wave curve and $U^{+}=\Psi_{4}\left(U_{3}, \hat{\varepsilon}\right)$ or $U_{3}=\tilde{\Psi}_{4}\left(U^{+},-\hat{\varepsilon}\right)$. Let

$$
\begin{aligned}
(2.3 .1) F\left(U_{1}, U^{+}, \varepsilon^{\text {in }}, \varepsilon^{\text {out }}, \hat{\varepsilon}\right) & =\Psi_{1 \frac{1}{2}}\left(\tilde{\Psi}_{3}\left(U_{1},-\varepsilon^{\text {in }}\right), \tilde{\Psi}_{3}\left(\tilde{\Psi}_{4}\left(U^{+},-\hat{\varepsilon}\right),-\varepsilon^{\text {out }}\right)\right) \\
& =\Psi_{1 \frac{1}{2}}\left(U_{0}, U_{2}\right)=0,
\end{aligned}
$$

then

$$
\frac{\partial F}{\partial\left(\varepsilon^{o u t}, \hat{\varepsilon}\right)}\left(U_{1}, U^{+}, 0,0,0\right)=\frac{\partial \Psi_{1 \frac{1}{2}}}{\partial\left(U_{l}, U_{r}\right)}\left(r_{3}\left(U_{3}\right), r_{4}\left(U^{+}\right)\right),
$$

where $r_{k}$ are the right eigenvectors of corresponding characteristic families. As $r_{3}$ and $r_{4}$ are independent, by the Implicit Function Theorem, there exits a unique solution for $(2.3 .1)$ and $U_{2}$ is given by $\tilde{\Psi}_{3}\left(\tilde{\Psi}_{4}\left(U^{+},-\hat{\varepsilon}\right),-\varepsilon^{o u t}\right)$.

We define the strength of a non-physical wave as the distance between its right and left states. Moreover,

we define the strength a phase boundary to be a fixed number $D$ which is bigger than all strengths of small waves.

The strength of a phase boundary will change slightly after it collides with a small physical wave such that the actual strength of a phase boundary should 
be $D$ plus an error term $O(\varepsilon)$ where $\varepsilon$ is the strength of the small wave. In what follows, one can see that this error term will be overwhelmed with $D$ in our analysis.

Definition 3.1 (Approaching Waves).

(i) We say that two small (possibly non-physical) fronts $\alpha$ and $\beta$, located at $x_{\alpha}<x_{\beta}$ and belonging to the characteristic family $k_{\alpha}, k_{\beta} \in\{1,2,3,4\}$ respectively, approach each other if and only if the two conditions hold:

- $x_{\alpha}$ and $x_{\beta}$ lie in the same set $\Omega^{-}, \Omega^{m}$ or $\Omega^{+}$.

- Either $k_{\alpha}>k_{\beta}$ or $k_{\alpha}=k_{\beta}$ and at least one of the waves is a genuinely nonlinear shock.

This case is represented by $(\alpha, \beta) \in \mathcal{A}$.

(ii) We say that a small wave $\alpha$ located at $x_{\alpha}$ is approaching a phase boundary at $x_{\beta}$ if and only if $k_{\alpha}<k_{\beta}$ and $x_{\alpha}>x_{\beta}$ or $k_{\alpha}>k_{\beta}$ and $x_{\alpha}<x_{\beta}$. This case is written as $\alpha \in \mathcal{A}_{b}$ if the $k_{\beta}$-wave is a backward phase boundary and $\alpha \in \mathcal{A}_{f}$ if the $k_{\beta}$-wave is a forward phase boundary.

Notice that the 2-family is linearly degenerate, such that a 2-wave (contact discontinuity) does not approach other 2-waves.

Definition Let $t>0$. The total weighted strength of waves in $U(t, \cdot)$ is defined by

$$
V(t)=\sum_{\alpha}\left|b_{\alpha}\right|
$$

where the summation ranges over all small wave fronts. The (weighted) wave interaction potentials are defined as

$$
\begin{gathered}
Q_{\mathcal{A}}(t)=\sum_{(\alpha, \beta) \in \mathcal{A}}\left|b_{\alpha} b_{\beta}\right|, \\
Q_{b}(t)=\sum_{\alpha \in \mathcal{A}_{b}}\left|b_{\alpha}\right|, \\
Q_{f}(t)=\sum_{\alpha \in \mathcal{A}_{f}}\left|b_{\alpha}\right|,
\end{gathered}
$$

and

$$
Q(t)=\kappa Q_{\mathcal{A}}(t)+Q_{b}(t)+Q_{f}(t) .
$$

The Glimm functional is

$$
\Gamma(t)=V(t)+\tilde{\kappa} Q(t)+\left|U^{*}(t)-U_{0}^{m}\right|,
$$

where $\kappa, \tilde{\kappa}>0$ are constants to be specified later. The vector $U^{*}(t)$ is the right state of the backward phase boundary at time $t$.

In order to prove the existence of the solution, we need the following interaction estimate.

Lemma 3.1. If a small wave $b_{\alpha}(\alpha=1,2,3)$ interacts with a phase boundary, producing outgoing waves $c_{1}, c_{2}$ and $c_{3}$. Then

$$
\sum_{k=1}^{3}\left|c_{k}\right|=O(1)\left|b_{\alpha}\right| .
$$


Proof. When $\alpha=1$ (or $\alpha=2$ ) and the phase boundary moves forward, this estimate is a direct consequence of Lemma 3.2 in [12] where we take $a_{1}=a_{2}=a_{3}=0$ and $b_{2}=0$ (or $b_{1}=0$, respectively). When $\alpha=1$ and the the phase boundary moves backward, this estimate is implied in Lemma 3.3 in [12] if we let $a_{1}=a_{2}=a_{3}=0$. Using Taylor expansions as in Lemma 3.2 and Lemma 3.3 in [12], we can show that (2.3.2) also holds for other cases.

We define for a given $\delta_{0}>0$ the domain

$$
\begin{aligned}
\tilde{\mathcal{D}}_{\delta_{0}}= & \text { cl }\left\{U: R \rightarrow R^{3} ; \text { there exist two points } x^{b}<x^{f} \text { in } R\right. \\
& \text { such that letting } \tilde{U}(x)= \begin{cases}U_{0}^{-}, & x<x^{b} \\
U_{0}^{m}, & x^{b}<x<x^{f} \\
U_{0}^{+}, & x>x^{f}\end{cases} \\
& \text { we have } \left.U-\tilde{U} \in L^{1}\left(R, R^{3}\right) \text { and T.V. }(U-\tilde{U}) \leq \delta_{0}\right\} .
\end{aligned}
$$

Lemma 3.2. We assume that the Finiteness Condition holds. There exist $\left\{w_{k}^{-}\right\},\left\{w_{k}^{m}\right\},\left\{w_{k}^{+}\right\}$, constants $\kappa, \tilde{\kappa}>0$ and $\delta>0$ such that the following holds

$\lim _{x \rightarrow-\infty} U(0, x)=U_{0}^{-}, \lim _{x \rightarrow \infty} U(0, x)=U_{0}^{+}$.

There exist points $x^{b}<x^{f}$ in $R$ such that

$$
U(x, 0) \in \begin{cases}\Omega^{-} & \text {for } x<x^{b} \\ \Omega^{m} & \text { for } x^{b}<x<x^{f} \\ \Omega^{+} & \text {for } x>x^{f} .\end{cases}
$$

If T.V. $(U(x, 0)-\tilde{U})<\delta$, then for any $t>0$ when two wave fronts $b_{\alpha}$ and $b_{\beta}$ interact we have

$$
\begin{aligned}
\Delta Q(t) & =Q(t+)-Q(t-) \\
& \leq \begin{cases}-c\left|b_{\alpha} b_{\beta}\right| & \text { if both waves are small } \\
-c\left|b_{\alpha}\right| & \text { if } \alpha \text { wave is small and } \beta \text { wave is a phase boundary },\end{cases}
\end{aligned}
$$

where the number $c$ is some small positive, uniform constant.

(ii) The same estimate holds for $\Delta \Gamma(t)=\Gamma(t+)-\Gamma(t-)$.

Proof. (i) Let $t>0$ be fixed time of interaction of two waves one of which could be a phase boundary or a non-physical wave.

- Case I Two small waves interact with each other.

By the standard estimates in [22], we have

$$
\begin{aligned}
\Delta Q_{b} & =O(1)\left|b_{\alpha} b_{\beta}\right|, \\
\Delta Q_{f} & =O(1)\left|b_{\alpha} b_{\beta}\right|, \\
\Delta Q_{\mathcal{A}} & =-\left|b_{\alpha} b_{\beta}\right|+O(1) V(t-)\left|b_{\alpha} b_{\beta}\right| .
\end{aligned}
$$

This is exactly Case I of Proposition3.4 in [17]. Let $C$ denote the largest uniform constant in the estimates above. If $\kappa \geq 4 C$ and $V(t) \leq 1 / \kappa$, one sees that (i) holds for $c=C$. 
- Case II A small wave interacts with the backward phase boundary from the left.

Suppose that the interaction is solved by the Accurate Riemann Solver and the outgoing waves are denoted by $c_{i}(i=1,2,3)$. If the small wave belongs to the 3 rd characteristic family, we have

$$
\begin{aligned}
\Delta Q_{b} & =-\left|b_{3}\right|, \\
\Delta Q_{f} & =\left|c_{3}\right|, \\
\Delta Q_{\mathcal{A}} & =O(1) V(t-)\left|b_{3}\right| .
\end{aligned}
$$

When $V(t-)<1 / 4 \kappa C$ and we choose a small weight $w_{3}^{m}$ for the transpassing wave $c_{3}$ such that $\left|c_{3}\right| \leq\left|b_{3}\right| / 4$, (i) holds for the constant $c=1 / 2$.

If the interaction is solved by the Approximate Riemann Solver, we have

$$
\begin{aligned}
\Delta Q_{b} & =-\left|b_{3}\right|, \\
\Delta Q_{f} & =\left|c_{3}\right|+\left|c_{4}\right|, \\
\Delta Q_{\mathcal{A}} & =O(1) V(t-)\left|b_{3}\right| .
\end{aligned}
$$

As the total strength of non-physical waves remains uniformly small [5], i.e. $\left|c_{4}\right|<$ $\left|b_{3}\right| / 4$, (i) holds if we choose $c=1 / 4$.

Similar assertion holds when the incoming small wave is a 2 -family wave and we need to choose a small weight $w_{2}^{m}$ for the transpassing wave $c_{2}$.

- Case III A small wave interacts with the forward phase boundary from the right.

This case is similar to Case II while we need to choose the weight $w_{1}^{m}$ (or $w_{2}^{m}$ ) sufficiently small if the incoming wave is a 1-wave (or 2-wave).

- Case IV A small wave interacts with a backward phase boundary from the right.

We need the Finiteness Condition in this case. Under the assumption that phase boundaries move much slower than a 1- or 3- wave, the small physical wave must be a 1 - wave and

$$
\begin{aligned}
\Delta Q_{b} & =-\left|b_{1}\right|, \\
\Delta Q_{f} & =\left|c_{3}\right|, \\
\Delta Q_{\mathcal{A}} & =O(1) V(t-)\left|b_{1}\right| .
\end{aligned}
$$

By the Finiteness Condition (2.2.1),

$$
\Delta Q(t) \leq(C \kappa V(t-)-1+\theta)\left|b_{1}\right| .
$$

Therefore, (i) holds for $c=(1-\theta) / 2$ if we choose $V(t-) \leq(1-\theta) / 2 \kappa C$.

- Case V A small wave interacts with a forward phase boundary from the left.

This case is similar to Case IV and we need the Finiteness Condition (2.2.2).

(ii) Note that

$$
\Delta V(t)=V(t+)-V(t-) \leq \begin{cases}C\left|b_{\alpha} b_{\beta}\right| & \text { in CaseI } \\ C\left|b_{\alpha}\right| & \text { in CaseII and CaseIII, }\end{cases}
$$


by [6] and Lemma 3.1. In cases I and III $U^{*}(t-)=U^{*}(t+)$, so $\left|U^{*}(t)-U_{0}^{m}\right|$ does not change across the interaction time $t$. In case II $\left|U^{*}(t-)-U^{*}(t+)\right|=O(1)\left|b_{\alpha}\right|$ by Lemma 3.1. Thus, if $\tilde{\kappa}$ is large enough, we get (ii) provided that

$$
V(t-) \leq \tilde{\delta}=\min \left\{\frac{1}{\kappa}, \frac{1}{4 C}, \frac{1-\theta}{2 \kappa C}\right\} .
$$

Notice that

$$
\begin{aligned}
V(t-) \leq & \Gamma(t-) \leq \Gamma(0)=V(0)+\tilde{\kappa} Q(0)+\left|U^{*}(0)-U_{0}^{m}\right| \\
\leq & C_{1} \cdot T \cdot V \cdot(U(0, \cdot)-\tilde{U})+\tilde{\kappa}\left\{\kappa C_{1} \cdot[T \cdot V \cdot(U(0, \cdot)-\tilde{U})]^{2}\right. \\
& \left.+2 C_{1} \cdot T \cdot V \cdot(U(0, \cdot)-\tilde{U})\right\}
\end{aligned}
$$

where $C_{1}$ is a uniform positive constant. If the constant $\delta$ is small enough, the inequality $T . V .(U(0, \cdot)-\tilde{U})<\delta$ implies $V(t-)<\tilde{\delta}$ and the result follows.

As in the case without the presence of large waves [5], Lemma 3.2 results in the following assertions. If $U(0, \cdot)$ satisfies the assumption of Lemma 3.2, then our wave front tracking algorithm generates a piecewise constant approximate solution that has finitely many discontinuity lines for all $t \in[0, \infty)$. Moreover, the functional $\Gamma$ is nonincreasing in time, and we have

$$
\begin{aligned}
\Gamma(t) & \leq \Gamma(0), \\
T . V \cdot(U(t, \cdot)-\hat{U}) & =O(1) \cdot \Gamma(t)=O(1) \cdot T \cdot V \cdot(U(t, \cdot)-\tilde{U})
\end{aligned}
$$

for some $\hat{U}$ in (2.3.3) as $\tilde{U}$. The total strength of all non-physical waves occurring at any fixed time $t>0$ is of the order $O(1)(\delta)$.

Following $[\mathbf{8}]$ and $[\mathbf{1 7}]$, we gather the main properties of the wave front tracking approximate solutions.

Theorem 3.3. Assume that a piecewise constant function $U(0, \cdot)$ satisfies the assumption of Lemma 3.2. Given $\varepsilon>0$, for some parameters $\delta>0$ the corresponding wave front tracking algorithm produces the function $U[0,+\infty) \mapsto$ $L^{1}\left(R ; R^{3}\right)$ such that:

(i) As a function of two variables, $U=U(t, x)$ is piecewise constant, with discontinuities occurring along finitely many lines in the $t-x$ plane. Only finitely many wave front interactions occur, each involving exactly two incoming fronts. Jumps can be of four types: shocks (or contact discontinuities), rarefactions, nonphysical waves and phase boundaries denoted as $\mathcal{F}=\mathcal{S} \cup \mathcal{R} \cup \mathcal{N P} \cup \mathcal{P B}$.

(ii) Along each shock (or contact discontinuity) $x=x_{\alpha}(t), \alpha \in \mathcal{S}$, the values $U^{-} \doteq U\left(t, x_{\alpha}-\right)$ and $U^{+} \doteq U\left(t, x_{\alpha}+\right)$ are related by

$$
U^{+}=S_{k_{\alpha}}\left(\sigma_{\alpha}\right)\left(U^{-}\right)
$$

for some $k_{\alpha} \in\{1,2,3\}$ and some wave size $\sigma_{\alpha}$. If the $k_{\alpha}$-family is genuinely nonlinear, then the entropy admissibility condition $\sigma_{\alpha}<0$ also holds. Moreover, the speed of the shock front satisfies

$$
\left|\dot{x}_{\alpha}-\lambda_{k_{\alpha}}\left(U^{+}, U^{-}\right)\right| \leq \varepsilon .
$$


(iii) Along each rarefaction front $x=x_{\alpha}(t), \alpha \in \mathcal{R}$, one has

$$
U^{+}=R_{k_{\alpha}}\left(\sigma_{\alpha}\right)\left(U^{-}\right), \quad \sigma_{\alpha} \in(0, \varepsilon]
$$

for some genuinely nonlinear family $k_{\alpha}$. Moreover,

$$
\left|\dot{x}_{\alpha}-\lambda_{k_{\alpha}}\left(U^{+}\right)\right| \leq \varepsilon .
$$

(iv) All non-physical fronts $x=x_{\alpha}(t), \alpha \in \mathcal{N} \mathcal{P}$ have the same speed:

$$
\dot{x}_{\alpha}(t) \equiv \hat{\lambda}
$$

where $\hat{\lambda}$ is a fixed constant strictly greater than all characteristic speeds. The total strength of all non-physical fronts in $U(t, \cdot)$ remains uniformly small, namely,

$$
\sum_{\alpha \in \mathcal{N} \mathcal{P}}\left|U\left(t, x_{\alpha}+\right)-U\left(t, x_{\alpha}-\right)\right| \leq \varepsilon \text { for all } t \geq 0 .
$$

(v) The backward and forward phase boundaries, denoted by $P_{1}$ and $P_{2}$ respectively, are determined by Theorem 2.3.

The function $U$ will be called an E-approximate solution of (2.1.1) and (2.1.3).

Now we can obtain the existence of the weak solution to (2.1.1) and (2.1.3). The following theorem is similar to the Theorem A in [17].

Theorem 3.4. If the Finiteness Condition is satisfied, then there exists $\delta_{0}>0$ such that for every $\bar{U} \in \tilde{\mathcal{D}}_{\delta_{0}}$ there exists a weak solution to (2.1.1) and (2.1.3) defined for all $t>0$.

Proof. Take $\bar{U} \in \tilde{\mathcal{D}}_{\delta_{0}}$, for some $\delta_{0}$ smaller than $\delta$ in Lemma 3.2. Given $\varepsilon>0$, fix a piecewise constant $\bar{U}_{\varepsilon} \in \tilde{\mathcal{D}}_{\delta_{0}}$, such that

$$
\left\|\bar{U}-\bar{U}_{\varepsilon}\right\|_{L^{1}\left(R, R^{3}\right)}<\varepsilon .
$$

Let $U_{\varepsilon}$ the the $\varepsilon$-approximation of $(2.1 .1)$ with $U_{\varepsilon}(0, \cdot)=\bar{U}_{\varepsilon}$, as in Theorem 3.3. Let $\varepsilon \rightarrow 0$, we can extract a sequence $U_{\varepsilon_{n}}$ converging in $L_{l o c}^{1}$ to a function $U(t, x)$. By the inequalities in Theorem 3.3, $U$ must be a solution to (2.1.1) and (2.1.3).

We can obtain the existence of the weak solutions by Theorem 3.4 under the Finiteness Condition. However, whether this condition holds has not been shown for any given system. In what follows we discuss a case where the Finiteness Condition is satisfied. Let us consider the initial value problem (2.1.1) and (2.1.3) given that the wave speeds $\sqrt{f_{v}}$ do not differ significantly between $\alpha$ and $\beta$-phase, i.e. $\left|\lambda_{1}\right| \approx\left|\lambda_{3}\right|$. This case is important in physics and the existence of the weak solutions is obtained in [12]. In the following theorem, we will show that the Finiteness Condition holds therefore the existence of weak solutions can be derived from Theorem 3.4 .

Corollary 3.5. Suppose that the wave speeds $\sqrt{f_{v}}$ do not differ significantly between $\alpha$ and $\beta$-phase, the conclusion in Theorem 3.4 holds.

Proof. We only need to show that the Finiteness Condition holds in this case.

Let us consider the wave interaction pattern shown in Fig2.2.1(a). Obviously, this is a special case of Lemma 3.3 in [12] with $a_{1}=a_{2}=a_{3}=0$ such that we have

$$
c_{3}=\delta b_{1} \text {, }
$$


where $c_{3}=\varepsilon_{3}^{\text {out }}$ and $b_{1}=\varepsilon_{1}^{\text {in }}$ for some constant $\delta$ satisfying $0<\delta<1$. When the incoming wave strength $\varepsilon_{1}^{i n}$ is very small,

$$
\frac{\varepsilon_{3}^{\text {out }}}{\varepsilon_{1}^{\text {in }}} \approx \frac{\partial \varepsilon_{3}^{\text {out }}}{\partial \varepsilon_{1}^{\text {in }}}=\left|m_{13}^{P_{1}}\right| .
$$

Choosing $w_{1}=w_{3}$, we have

$$
\frac{w_{3}}{w_{1}}\left|m_{13}^{P_{1}}\right| \approx \delta<1 .
$$

Therefore, we have (2.2.1) for some number $\theta$ satisfying $\delta<\theta<1$.

Similarly, considering the wave interaction pattern shown in Fig2.2.1(b), we can see that (2.2.2) holds for $w_{1}=w_{3}$.

\subsection{The Lyapunov Functional and Stability}

In order to show the $L^{1}$ stability of the weak solutions, we follow $[\mathbf{1 8}, \mathbf{1 9}, \mathbf{8}]$ and [17] to introduce the Lyapunov functional $\Phi(U, V)$ satisfying

$$
\frac{1}{C} \cdot\|U(t, \cdot)-V(t, \cdot)\|_{L^{1}} \leq \Phi(U(t, \cdot), V(t, \cdot)) \leq C \cdot\|U(t, \cdot)-V(t, \cdot)\|_{L^{1}}
$$

and

$$
\Phi(U(t, \cdot), V(t, \cdot))-\Phi(U(s, \cdot), V(s, \cdot)) \leq C \cdot \varepsilon \cdot(t-s) \quad \forall t>s \geq 0,
$$

for any two $\varepsilon$-approximate solutions $U$ and $V$. The functional is equivalent to the $L^{1}$ distance between $U$ and $V$ and is "almost decreasing" in time.

We define

$$
\Phi(U, V) \doteq \sum_{k \in I} \int_{-\infty}^{+\infty}\left|q_{k}(x)\right| W_{k}(x) d x,
$$

where $I=\left\{1,1 \frac{1}{2}, 2,2 \frac{1}{2}, 3\right\}$ is set of the indices without non-physical waves and $q_{k}$ is the size of the $k$-th shock. The weights $W_{k}$ are defined by

$$
W_{k}(x) \doteq 1+\kappa_{1} A_{k}(x)+\kappa_{2}[Q(u)+Q(v)] .
$$

The constant $\kappa_{1}$ and $\kappa_{2}$ are to be defined later. $Q$ is the Glimm's interaction functional. When $k \in\left\{1 \frac{1}{2}, 2,2 \frac{1}{2}\right\}$ we simply take

$$
A_{k} \doteq\left[\sum_{x_{\alpha}<x, k<k_{\alpha} \leq 3}+\sum_{x_{\alpha}>x, 1 \leq k_{\alpha}<k}\right]\left|\varepsilon_{\alpha}\right| .
$$

The summations here extend to waves both of $U$ and $V$. This is similar to the linearly degenerate case in [17]. When $k \in\{1,3\}, A_{k}=B_{k}+C_{k}$ where

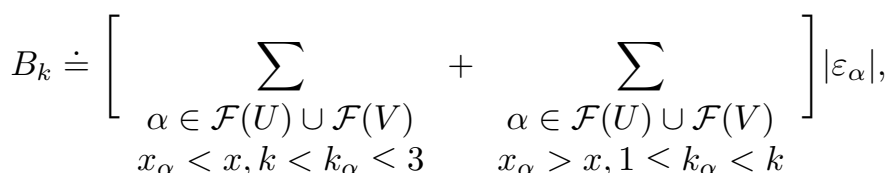




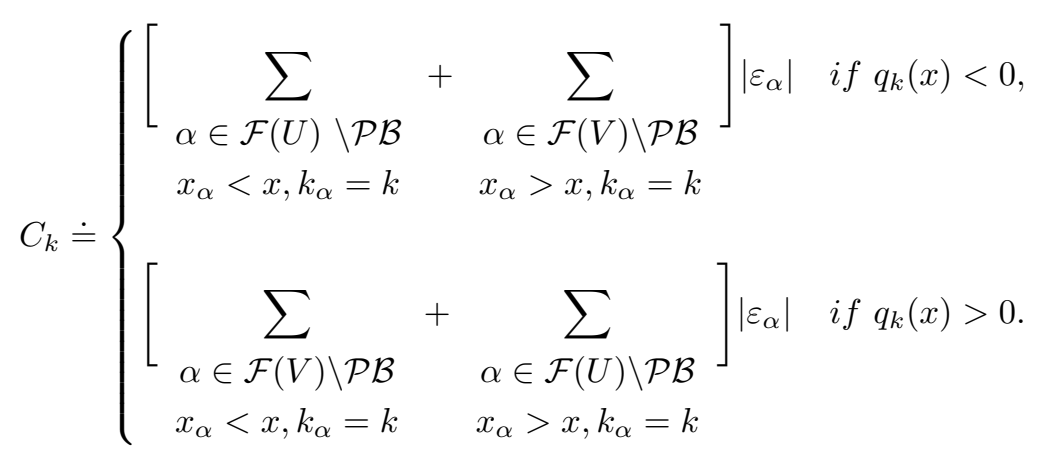

We can always assume that

$$
1 \leq W_{k}(x) \leq 2
$$

given that $W_{k}(x)$ does not contain any phase boundaries and

$$
1+m \kappa_{1} D \leq W_{k}(x) \leq 2+m \kappa_{1} D
$$

if $W_{k}(x)$ contains $m$ phase boundaries. As there are in total 4 phase boundaries in both $U$ and $V$, we have $m \leq 4$ and

$$
1 \leq W_{k}(x) \leq 2+4 \kappa_{1} D .
$$

Thus,

$$
\frac{1}{2+4 \kappa_{1} D}|U(x)-V(x)| \leq \sum_{k \in I}\left|q_{k}(x)\right| \leq\left(2+4 \kappa_{1} D\right)|U(x)-V(x)|
$$

and (2.4.1) holds.

Theorem 4.1. If the Stability Condition is satisfied, there exists $\delta_{0}>0, L>0$, a closed domain $\mathcal{D}_{\delta_{0}} \subset L_{\text {loc }}^{1}\left(R, R^{3}\right)$ containing $\tilde{\mathcal{D}}_{\delta_{0}}$, and a continuous semigroup $S:[0,+\infty) \times \mathcal{D}_{\delta_{0}} \rightarrow \mathcal{D}_{\delta_{0}}$ such that

(i) $S(0, \bar{U})=\bar{U}, S(t+s)=S(t, S(s, \bar{U})) \quad \forall t, s \geq 0 \forall \bar{U} \in \mathcal{D}_{\delta_{0}}$,

(ii) $\|S(t, \bar{U})-S(s, \bar{V})\|_{L^{1}} \leq L\left(|t-s|+\|\bar{U}-\bar{V}\|_{L^{1}} \quad \forall t, s \geq 0 \forall \bar{U}, \bar{V} \in \mathcal{D}_{\delta_{0}}\right.$.

(iii) Each trajectory $t \longmapsto S(t, \bar{U})$ is a weak solution of (2.1.1),(2.1.3).

Proof. This is a standard proof in $[\mathbf{8}, \mathbf{1 7}]$ given that (2.4.1) and (2.4.2) hold.

Theorem 4.1 shows the stability of the weak solution under the Stability Condition, while it remains unknown whether this condition holds in any given system. Similar to Corollary 3.5, we consider the case where the wave speeds $\sqrt{f_{v}}$ do not differ significantly between $\alpha$ and $\beta$-phase. We will show that the Stability Condition also holds in this case and the $L^{1}$ stability of the weak solution can be obtained consequently.

Corollary 4.2. Suppose that the wave speeds $\sqrt{f_{v}}$ do not differ significantly between $\alpha$ and $\beta$-phase, i.e. $\left|\lambda_{1}\right| \approx\left|\lambda_{3}\right|$, the conclusions in Theorem 4.1 hold.

Proof. We only need to verify that the Stability Condition holds in this case. 
Firstly, we consider the wave interaction pattern shown in Fig 2.2.1(a). We know from the assumption that

$$
\lambda_{3}=-\lambda_{1}+\varepsilon_{0},
$$

where $\lambda_{1}<0$ and $\lambda_{3}>0$ are the characteristic speeds of the 1 and 3 -wave, respectively, and $\varepsilon_{0}$ is constant satisfying that $\left|\varepsilon_{0}\right| \ll\left|\lambda_{i}\right|(i=1,3)$. Hence,

$$
\begin{aligned}
&\left|\frac{\lambda_{3}-\sigma_{P_{1}}}{\lambda_{1}-\sigma_{P_{1}}}\right|=\left|\frac{-\lambda_{1}+\varepsilon_{0}-\sigma_{P_{1}}}{\lambda_{1}-\sigma_{P_{1}}}\right|=\left|\frac{\lambda_{1}-\sigma_{P_{1}}-\varepsilon+2 \sigma_{P_{1}}}{\lambda_{1}-\sigma_{P_{1}}}\right| \\
&=\left|1+\frac{2 \sigma_{P_{1}}-\varepsilon}{\lambda_{1}-\sigma_{P_{1}}}\right| \leq 1+\left|\frac{2 \sigma_{P_{1}}-\varepsilon}{\lambda_{1}-\sigma_{P_{1}}}\right| \leq 1+O\left(\left|\sigma_{P_{1}}\right|+|\varepsilon|\right),
\end{aligned}
$$

where the last inequality holds because the phase boundary moves much slower than a 1 or 3 -wave, i.e. $\left|\lambda_{1}\right| \gg\left|\sigma_{P_{1}}\right|$, in our problem.

Choosing the weights $\tilde{w}_{1}=\tilde{w}_{3}$, we have

$$
\frac{\tilde{w}_{3}}{\tilde{w}_{1}}\left|m_{13}^{P_{1}}\right|\left|\frac{\lambda_{3}-\sigma_{P_{1}}}{\lambda_{1}-\sigma_{P_{1}}}\right|=\left|m_{13}^{P_{1}}\right|\left(1+O\left(\left|\sigma_{P_{1}}\right|+|\varepsilon|\right)\right) \approx \frac{\partial \varepsilon_{3}^{\text {out }}}{\partial \varepsilon_{1}^{\text {in }}} \approx \frac{\varepsilon_{3}^{\text {out }}}{\varepsilon_{1}^{\text {in }}}=\delta<1,
$$

where $\delta$ is the same as in Corollary 3.5 and last inequality is derived from Lemma 3.3 in [?]. Threrefore, (2.2.3) holds if we choose some number $\Theta$ satisfying $\delta<\Theta<1$. Similarly, (2.2.4) holds with $\tilde{w}_{1}=\tilde{w}_{3}$ if we consider the wave interaction pattern shown in Fig 2.2.1(b).

In order to prove (2.4.2), we differentiate the functional $\Phi$ at a time $t$ which is not the interaction time of the waves in $U(t, \cdot)$ or $V(t, \cdot)$ to show that

$$
\begin{aligned}
\frac{d}{d t} \Phi(U(t), V(t))= & \sum_{\alpha \in \mathcal{F}} \sum_{k \in I}\left\{\left|q_{k}\left(x_{\alpha}-\right)\right| W_{k}\left(x_{\alpha}-\right)-\left|q_{k}\left(x_{\alpha}+\right)\right| W\left(x_{\alpha}+\right)\right\} \dot{x}_{\alpha} \\
= & \sum_{\alpha \in \mathcal{F}} \sum_{k \in I}\left\{\left|q_{k}\left(x_{\alpha}+\right)\right| W\left(x_{\alpha}+\right)\left(\lambda_{k}\left(x_{\alpha}+\right)-\dot{x}_{\alpha}\right)\right. \\
& \left.-\left|q_{k}\left(x_{\alpha}-\right)\right| W\left(x_{\alpha}-\right)\left(\lambda_{k}\left(x_{\alpha}-\right)-\dot{x}_{\alpha}\right)\right\} \dot{x}_{\alpha},
\end{aligned}
$$

where $\dot{x}_{\alpha}$ is the speed of the discontinuity at the $\alpha$ wave. Let

$$
E_{\alpha, k}=\left|q_{k}^{\alpha+}\right| W_{k}^{\alpha+}\left(\lambda_{k}^{\alpha+}-\dot{x}_{\alpha}\right)-\left|q_{k}^{\alpha-}\right| W_{k}^{\alpha-}\left(\lambda_{k}^{\alpha-}-\dot{x}_{\alpha}\right),
$$

where $q_{k}^{\alpha+}=q_{k}\left(x_{\alpha}+\right), \lambda_{k}^{\alpha+}=\lambda_{k}\left(x_{\alpha}+\right)$ and so on. Then (2.4.7) becomes

$$
\frac{d}{d t} \Phi(U(t), V(t))=\sum_{\alpha \in \mathcal{F}} \sum_{k \in I} E_{\alpha, k} .
$$

Our main goal will be to establish

$$
\begin{gathered}
\sum_{k \in I} E_{\alpha, k}=O(1) \cdot\left|\varepsilon_{\alpha}\right| \quad \forall \alpha \in \mathcal{N} \mathcal{P}, \\
\sum_{k \in I} E_{\alpha, k} \leq 0 \quad \forall \alpha \in \mathcal{P B}, \\
\sum_{k \in I} E_{\alpha, k}=O(1) \cdot \varepsilon\left|\varepsilon_{\alpha}\right| \quad \forall \alpha \in \mathcal{S} \cup \mathcal{R} .
\end{gathered}
$$



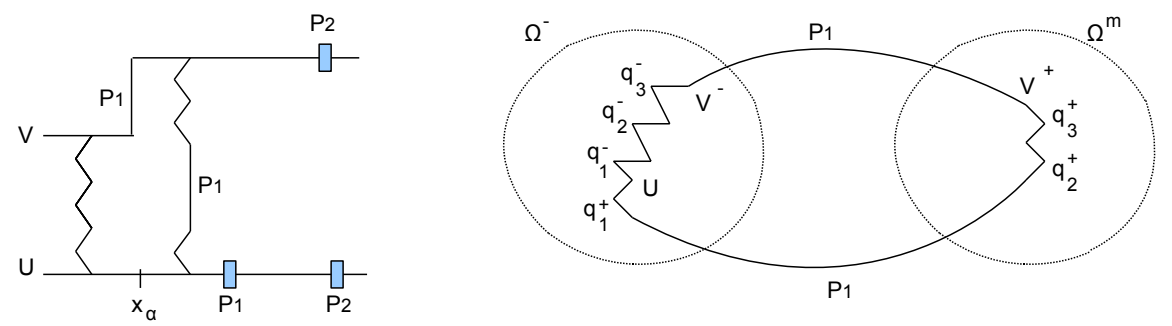

Figure 2.5.1. Case 1

Also we need that all weights $W_{k}(x)$ decrease after an interaction of wave fronts in $U$ or $V$. Recalling Lemma 3.1 and Lemma 3.2(i). One sees this statement holds if $\kappa_{2} \gg \kappa_{1}$ in (2.4.3).

The proof of $(2.4 .8)$ is the same as $[\mathbf{8}, \mathbf{1 7}]$ and thus omitted. In next section, we will show that (2.4.9) and (2.4.10) hold in different wave interaction patterns. Combining (2.4.8), (2.4.9) and (2.4.10), recalling (2.3.9) and the uniform bound on the total strengths of waves, denoted by the functional $\Gamma(t)$, we have

$$
\frac{d}{d t} \Phi(U(t), V(t)) \leq C \cdot \varepsilon
$$

Integrating this inequality gives (2.4.2).

\subsection{Stability of Approximate Solution}

In this section, we will prove (2.4.9) and (2.4.10). In what follows, we drop the notation $\alpha$ to write $E_{\alpha, k}$ as

$$
E_{k}=\left|q_{k}^{+}\right| W_{k}^{+}\left(\lambda_{k}^{+}-\dot{x}_{\alpha}\right)-\left|q_{k}^{-}\right| W_{k}^{-}\left(\lambda_{k}^{-}-\dot{x}_{\alpha}\right)
$$

without any ambiguity. We will need to choose different weights $\tilde{w}_{k}(k \in\{1,2,3\})$ in proving (2.4.9) and (2.4.10). In general, all weights will be chosen very small while some should be relatively larger than others. We summarize the sizes of the weights as follows:

(i) In the domain $\Omega^{-}, \tilde{w}_{1}^{-}$is small relative to $\tilde{w}_{2}^{-}$and $\tilde{w}_{3}^{-}$;

(ii) In the domain $\Omega^{+}, \tilde{w}_{3}^{+}$is small relative to $\tilde{w}_{1}^{+}$and $\tilde{w}_{2}^{+}$;

(iii) All the weights in the domain $\Omega^{m}$ are small. Moreover, $\tilde{w}_{1}^{m}$ and $\tilde{w}_{3}^{m}$ need to satisfy (2.2.3) and (2.2.4).

2.5.1. Cases of Phase Boundaries-The Estimate (2.4.9). We consider the cases where the discontinuities in $U$ or $V$ are phase boundaries. Under the assumption that a phase boundary moves much slower than a 1 or 3 - wave, we have in total four different cases.

Case 1 See Fig 2.5.1. 
As there is no phase boundary in the solution of the Riemann problem $\left(U, V^{-}\right)$, $q_{1 \frac{1}{2}}^{-}=0$ and

$$
E_{1 \frac{1}{2}}=\left|q_{1 \frac{1}{2}}^{+}\right| W_{1 \frac{1}{2}}^{+}\left(\lambda_{1 \frac{1}{2}}^{+}-\dot{x}_{\alpha}\right) .
$$

Therefore, (2.4.4) implies that

$$
E_{1 \frac{1}{2}} \leq D \cdot 4\left|\sigma_{P_{1}}\right|
$$

where $\left|q_{1 \frac{1}{2}}^{+}\right|=D$ is the strength of the phase boundary.

Notice that $\lambda_{1}^{ \pm}<0$, we can always assume that $\lambda_{1}^{ \pm}-\dot{x}_{\alpha}<-c<0$ due to the fact that the speed of a phase boundary $\left|\dot{x_{\alpha}}\right|=\left|\sigma_{P_{1}}\right|$ is very small compared with characteristic speeds $\left|\lambda_{k}^{ \pm}\right|(k=1,3)$. Thus,

$$
\begin{aligned}
E_{1}= & \left|q_{1}^{+}\right|\left(W_{1}^{+}-W_{1}^{-}\right)\left(\lambda_{1}^{+}-\dot{x}_{\alpha}\right) \\
& +W_{1}^{-}\left[\left|q_{1}^{+}\right|\left(\lambda_{1}^{+}-\dot{x}_{\alpha}\right)-\left|q_{1}^{-}\right|\left(\lambda_{1}^{-}-\dot{x}_{\alpha}\right)\right] \\
\leq & -\left|q_{1}^{+}\right| \cdot \kappa_{1} D \cdot\left|\lambda_{1}^{+}-\dot{x}_{\alpha}\right|+2\left|q_{1}^{-}\right| \cdot\left|\lambda_{1}^{-}-\dot{x}_{\alpha}\right|,
\end{aligned}
$$

where $W_{1}^{+}-W_{1}^{-}=\kappa_{1} D$. The 2 -family wave is a contact discontinuity whose characteristic speed, $\lambda_{2}^{ \pm}$, is always 0 in Lagrange coordinates. Such that

$$
\begin{aligned}
E_{2} & =\left(\left|q_{2}^{-}\right| W_{2}^{-}-\left|q_{2}^{+}\right| W_{2}^{+}\right) \dot{x}_{\alpha} \\
& \leq-2 \kappa_{1} D\left|q_{2}^{-}\right| \cdot\left|\dot{x}_{\alpha}\right|+\left(\kappa_{1} D+2\right) \cdot\left|q_{2}^{+}\right| \cdot\left|\dot{x}_{\alpha}\right|,
\end{aligned}
$$

where the inequality holds because $1+2 \kappa_{1} D \leq W_{2}^{-} \leq 2+2 \kappa_{1} D$ and $1+\kappa_{1} D \leq$ $W_{2}^{+} \leq 2+\kappa_{1} D$ by (2.4.5). Notice that $\lambda_{3}^{ \pm}-\dot{x}_{\alpha}>0$, we have

$$
\begin{aligned}
E_{3} & =\left|q_{3}^{+}\right| W_{3}^{+}\left(\lambda_{3}^{+}-\dot{x}_{\alpha}\right)-\left|q_{3}^{-}\right| W_{3}^{-}\left(\lambda_{3}^{-}-\dot{x}_{\alpha}\right) \\
& \leq\left(3 \kappa_{1} D+2\right) \cdot\left|q_{3}^{+}\right| \cdot\left|\lambda_{3}^{+}-\dot{x}_{\alpha}\right|-4 \kappa_{1} D \cdot\left|q_{3}^{-}\right| \cdot\left|\lambda_{3}^{-}-\dot{x}_{\alpha}\right| .
\end{aligned}
$$

Summing (2.5.1), (2.5.2) and (2.5.3) gives

$$
\begin{aligned}
\sum_{k=1,1 \frac{1}{2}, 2,3} E_{k} \leq & 4 D \cdot\left|\sigma_{P_{1}}\right|-\kappa_{1} D \cdot\left|q_{1}^{+}\right| \cdot\left|\lambda_{1}^{+}-\dot{x}_{\alpha}\right| \\
& -2 \kappa_{1} D\left|q_{2}^{-}\right| \cdot\left|\dot{x}_{\alpha}\right|+\kappa_{1} D \cdot\left|q_{2}^{+}\right| \cdot\left|\dot{x}_{\alpha}\right| \\
& +3 \kappa_{1} D\left|q_{3}^{+}\right| \cdot\left|\lambda_{3}^{+}-\dot{x}_{\alpha}\right|-4 \kappa_{1} D \cdot\left|q_{3}^{-}\right| \cdot\left|\lambda_{3}^{-}-\dot{x}_{\alpha}\right| \\
& +2\left|q_{1}^{-}\right| \cdot\left|\lambda_{1}^{-}-\dot{x}_{\alpha}\right|+2\left|q_{3}^{+}\right| \cdot\left|\lambda-\dot{x}_{\alpha}\right|+2\left|q_{2}^{+}\right| \cdot\left|\dot{x}_{\alpha}\right| .
\end{aligned}
$$

Similar to Lemma 5.1(iv) in [17], we have the following lemma.

Lemma 5.1. In case1, we have

$$
\left|\varepsilon_{2}^{+}\right|+\left|\varepsilon_{3}^{+}\right| \leq O(1)\left(\left|\varepsilon_{2}^{-}\right|+\left|\varepsilon_{3}^{-}\right|\right),
$$

where $\varepsilon_{k}^{ \pm}$are the unweighted strengths of corresponding waves.

Proof. If $\varepsilon_{2}^{-}=\varepsilon_{3}^{-}=0$, by the uniqueness of the solution to the Riemann problem $\left(U, V^{+}\right), \varepsilon_{2}^{+}=\varepsilon_{3}^{+}=0$. Then the result follows from the Lipschitz continuity of the problem. 

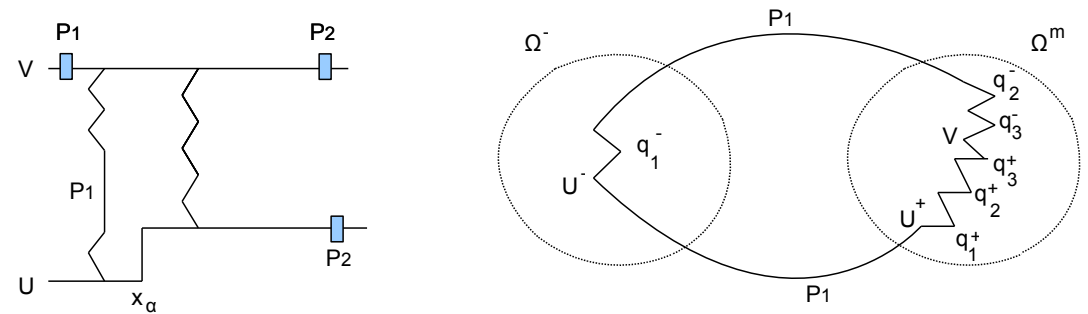

Figure 2.5.2. Case 2.

If we choose the constant $\kappa_{1}$ very large and the weights $\tilde{w}_{2}^{-}$and $\tilde{w}_{3}^{-}$big enough, then (2.4.9) holds by Lemma 5.1.

\section{Case 2 See Fig2.5.2.}

As there is no phase boundary in the solution of the Riemann problem $\left(U^{+}, V\right)$, $q_{1 \frac{1}{2}}^{+}=0$ and

$$
E_{1 \frac{1}{2}}=-\left|q_{1 \frac{1}{2}}^{-}\right| W_{1 \frac{1}{2}}^{-}\left(\lambda_{1 \frac{1}{2}}^{-}-\dot{x}_{\alpha}\right) \leq D \cdot 4\left|\sigma_{P_{1}}\right|
$$

Moreover, we have

$$
\begin{aligned}
E_{1} & =\left|q_{1}^{+}\right| W_{1}^{+}\left(\lambda_{1}^{+}-\dot{x}_{\alpha}\right)-\left|q_{1}^{-}\right| W_{1}^{-}\left(\lambda_{1}^{-}-\dot{x}_{\alpha}\right) \\
& \leq-2 \kappa_{1} D \cdot\left|q_{1}^{+}\right| \cdot\left|\lambda_{1}^{+}-\dot{x}_{\alpha}\right|+\left(\kappa_{1} D+2\right) \cdot\left|q_{1}^{-}\right| \cdot\left|\lambda_{1}^{-}-\dot{x}_{\alpha}\right|,
\end{aligned}
$$

and

$$
\begin{aligned}
E_{2} & =\left(\left|q_{2}^{-}\right| W_{2}^{-}-\left|q_{2}^{+}\right| W_{2}^{+}\right) \dot{x}_{\alpha} \\
& =\left[\left|q_{2}^{-}\right|\left(W_{2}^{-}-W_{2}^{+}\right)+W_{2}^{+}\left(\left|q_{2}^{-}\right|-\left|q_{2}^{+}\right|\right)\right] \dot{x}_{\alpha} \\
& \leq-\kappa_{1} D\left|q_{2}^{-}\right| \cdot\left|\dot{x}_{\alpha}\right|+2\left|q_{2}^{+}\right| \cdot\left|\dot{x}_{\alpha}\right|,
\end{aligned}
$$

where $1 \leq W_{2}^{+} \leq 2$ by (2.4.4) as $W_{2}^{+}$does not contain any phase boundaries. We also have

$$
\begin{aligned}
E_{3} & =\left|q_{3}^{+}\right| W_{3}^{+}\left(\lambda_{3}^{+}-\dot{x}_{\alpha}\right)-\left|q_{3}^{-}\right| W_{3}^{-}\left(\lambda_{3}^{-}-\dot{x}_{\alpha}\right) \\
& \leq\left(2 \kappa_{1} D+2\right)\left|q_{3}^{+}\right| \cdot\left|\lambda_{3}^{+}-\dot{x}_{\alpha}\right|-3 \kappa_{1} D \cdot\left|q_{3}^{-}\right| \cdot\left|\lambda_{3}^{-}-\dot{x}_{\alpha}\right|
\end{aligned}
$$



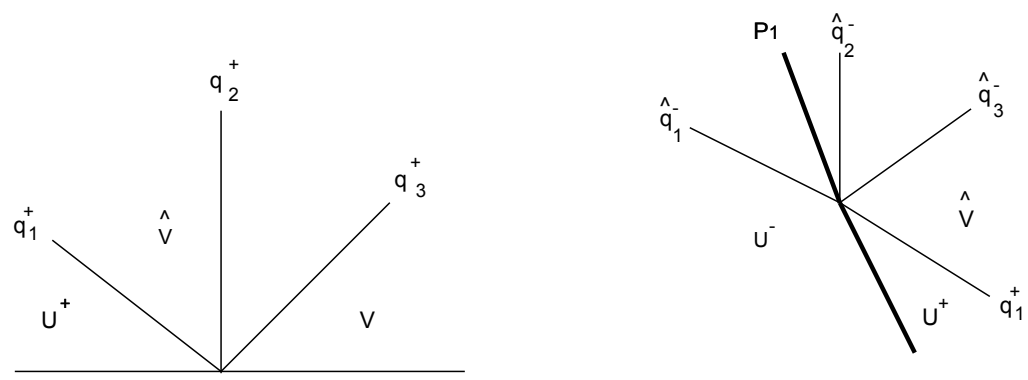

Figure 2.5.3. The definition of $\hat{V}$.

Summing up (2.5.5) through (2.5.8) gives

$$
\begin{aligned}
(2.5 .9) \sum_{k=1,1 \frac{1}{2}, 2,3} E_{k} \leq & -2 \kappa_{1} D \cdot\left|q_{1}^{+}\right| \cdot\left|\lambda_{1}^{+}-\dot{x}_{\alpha}\right|+\kappa_{1} D \cdot\left|q_{1}^{-}\right| \cdot\left|\lambda_{1}^{-}-\dot{x}_{\alpha}\right| \\
& -\kappa_{1} D\left|q_{2}^{-}\right| \cdot\left|\dot{x}_{\alpha}\right|+2 \kappa_{1} D\left|q_{3}^{+}\right| \cdot\left|\lambda_{3}^{+}-\dot{x}_{\alpha}\right| \\
& -3 \kappa_{1} D \cdot\left|q_{3}^{-}\right| \cdot\left|\lambda_{3}^{-}-\dot{x}_{\alpha}\right|+4 D\left|\sigma_{P_{1}}\right|+2\left|q_{1}^{-}\right| \cdot\left|\lambda_{1}^{-}-\dot{x}_{\alpha}\right| \\
& +2\left|q_{2}^{+}\right| \cdot\left|\dot{x}_{\alpha}\right|+2\left|q_{3}^{+}\right| \cdot\left|\lambda_{3}^{+}-\dot{x}_{\alpha}\right| .
\end{aligned}
$$

When $q_{2}^{+}=q_{3}^{+}=0,(2.4 .9)$ holds for a large $\kappa_{1}$ and a small weight $\tilde{w}_{1}^{-}$. When $q_{2}^{+}, q_{3}^{+} \neq 0$, we define $\hat{V}=S_{1}\left(U^{+}, q_{1}^{+}\right)$to be the state connected to $U$ on the right through a 1-shock (See Fig2.5.3).

Lemma 5.2. For the states $U^{-}, U^{+}, V, \hat{V}$ and the waves $q_{i}^{+}$and $\hat{q}_{i}^{-}(i=1,2,3)$ defined in Fig2.5.3, we have the following estimates

$$
\begin{aligned}
& q_{1}^{-}=\hat{q}_{1}^{-}+R_{2}, \\
& q_{2}^{-}=\hat{q}_{2}^{-}+q_{2}^{+}+R_{2}, \\
& q_{3}^{-}=\hat{q}_{3}^{-}+q_{3}^{+}+R_{2},
\end{aligned}
$$

where $R_{2}=O\left(\left|\hat{q}_{3}^{-}\right|\left(\left|q_{2}^{+}\right|+\left|q_{3}^{+}\right|\right)\right)$is a second order error term.

Proof. In the wave interaction pattern $\left(U^{-}, \hat{V}\right)+(\hat{V}, V) \longrightarrow\left(U^{-}, V\right)$ in Fig2.5.4, $\hat{q}_{3}^{-}$approaches $q_{2}^{+}$and $q_{3}^{+}$. Such that the lemma holds by Theorem 19.2 in $[22]$.

Notice that 


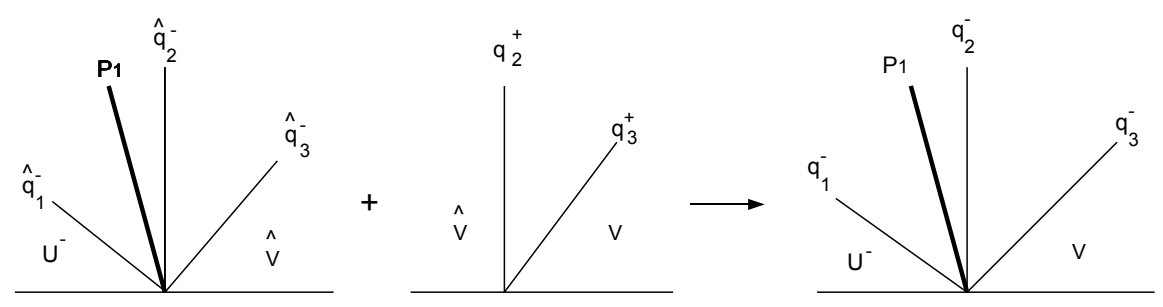

Figure 2.5.4. The wave interaction pattern $\left(U^{-}, \hat{V}\right)+$ $(\hat{V}, V) \longrightarrow\left(U^{-}, V\right)$.

$$
\begin{gathered}
2 \kappa_{1} D\left|q_{3}^{-}-\hat{q}_{3}^{-}\right| \cdot\left|\lambda_{3}^{+}-\dot{x}_{\alpha}\right|-3 \kappa_{1} D \cdot\left|q_{3}^{-}\right| \cdot\left|\lambda_{3}^{-}-\dot{x}_{\alpha}\right| \\
\leq 2 \kappa_{1} D\left(\left|q_{3}^{-}\right|+\left|\hat{q}_{3}^{-}\right|\right) \cdot\left|\lambda_{3}^{+}-\dot{x}_{\alpha}\right|-3 \kappa_{1} D \cdot\left|q_{3}^{-}\right| \cdot\left|\lambda_{3}^{-}-\dot{x}_{\alpha}\right| \\
=-\kappa_{1} D \cdot\left|q_{3}^{-}\right| \cdot\left|\lambda_{3}^{-}-\dot{x}_{\alpha}\right|+2 \kappa_{1} D\left|\hat{q}_{3}^{-}\right| \cdot\left|\lambda_{3}^{+}-\dot{x}_{\alpha}\right| \\
+2 \kappa_{1} D\left|q_{3}^{-}\right| \cdot\left(\left|\lambda_{3}^{+}-\dot{x}_{\alpha}\right|-\left|\lambda_{3}^{-}-\dot{x}_{\alpha}\right|\right) \\
\leq \quad-\kappa_{1} D \cdot\left|q_{3}^{-}\right| \cdot\left|\lambda_{3}^{-}-\dot{x}_{\alpha}\right|+2 \kappa_{1} D\left|\hat{q}_{3}^{-}\right| \cdot\left|\lambda_{3}^{+}-\dot{x}_{\alpha}\right|+2 \kappa_{1} D\left|q_{3}^{-}\right| \cdot\left|\lambda_{3}^{+}-\lambda_{3}^{-}\right| \\
\leq \quad-\kappa_{1} D \cdot\left|q_{3}^{-}\right| \cdot\left|\lambda_{3}^{-}-\dot{x}_{\alpha}\right|+2 \kappa_{1} D\left|\hat{q}_{3}^{-}\right| \cdot\left|\lambda_{3}^{+}-\dot{x}_{\alpha}\right|+2 \kappa_{1} D\left|q_{3}^{-}\right| \cdot O\left(\left|\hat{q}_{3}^{-}\right|\right),
\end{gathered}
$$

where the last inequality holds because $\left|\lambda_{3}^{+}-\lambda_{3}^{-}\right|=O\left(\left|q_{3}^{+}-q_{3}^{-}\right|\right)=O\left(\left|\hat{q}_{3}^{-}\right|\right)$. Thus, (2.5.9) becomes

$$
\begin{aligned}
\sum_{k=1,1 \frac{1}{2}, 2,3} E_{k} \leq & -2 \kappa_{1} D \cdot\left|q_{1}^{+}\right| \cdot\left|\lambda_{1}^{+}-\dot{x}_{\alpha}\right|+\kappa_{1} D \cdot\left|\hat{q}_{1}^{-}\right| \cdot\left|\lambda_{1}^{-}-\dot{x}_{\alpha}\right| \\
& -\kappa_{1} D\left|q_{2}^{-}\right| \cdot\left|\dot{x}_{\alpha}\right|-\kappa_{1} D \cdot\left|q_{3}^{-}\right| \cdot\left|\lambda_{3}^{-}-\dot{x}_{\alpha}\right| \\
& +2 \kappa_{1} D\left|\hat{q}_{3}^{-}\right| \cdot\left|\lambda_{3}^{+}-\dot{x}_{\alpha}\right|+2 \kappa_{1} D \cdot O(1)\left(\left|q_{3}^{-}\right| \cdot\left|\hat{q}_{3}^{-}\right|\right) \\
& +O\left(\left|\sigma_{P_{1}}\right|+\left|q_{1}^{-}\right|+\left|q_{2}^{+}\right|+\left|q_{3}^{+}\right|\right)+R_{2} .
\end{aligned}
$$

By the Stability Condition (2.2.3), we have in the wave interaction pattern shown in Fig2.5.3(b) that there exist weights $\tilde{w}_{1}^{+}$and $\hat{w}_{3}^{-}$such that $\left|\hat{q}_{3}^{-}\right| \cdot\left|\lambda_{3}^{+}-\dot{x}_{\alpha}\right| \leq$ $\Theta\left|q_{1}^{+}\right| \cdot\left|\lambda_{1}^{+}-\dot{x}_{\alpha}\right|$. Thus,

$$
\begin{aligned}
\sum_{k=1,1 \frac{1}{2}, 2,3} E_{k} \leq & -2(1-\Theta) \kappa_{1} D \cdot\left|q_{1}^{+}\right| \cdot\left|\lambda_{1}^{+}-\dot{x}_{\alpha}\right|+\kappa_{1} D \cdot\left|\hat{q}_{1}^{-}\right| \cdot\left|\lambda_{1}^{-}-\dot{x}_{\alpha}\right| \\
& -\kappa_{1} D\left|q_{2}^{-}\right| \cdot\left|\dot{x}_{\alpha}\right|-\kappa_{1} D \cdot\left|q_{3}^{-}\right| \cdot\left|\lambda_{3}^{-}-\dot{x}_{\alpha}\right|+O(1),
\end{aligned}
$$

where $O(1)=2 \kappa_{1} D \cdot O(1)\left(\left|q_{3}^{-}\right| \cdot\left|\hat{q}_{3}^{-}\right|\right)+O\left(\left|\sigma_{P_{1}}\right|+\left|q_{1}^{-}\right|+\left|q_{2}^{+}\right|+\left|q_{3}^{+}\right|\right)+R_{2}$ which will be overwhelmed by other terms if we choose $\kappa_{1}$ very large. Then (2.4.9) holds if we choose $\kappa_{1}$ large and $\tilde{w}_{1}^{-}$very small relative to other weights.

Case 3 See Fig2.5.5. 

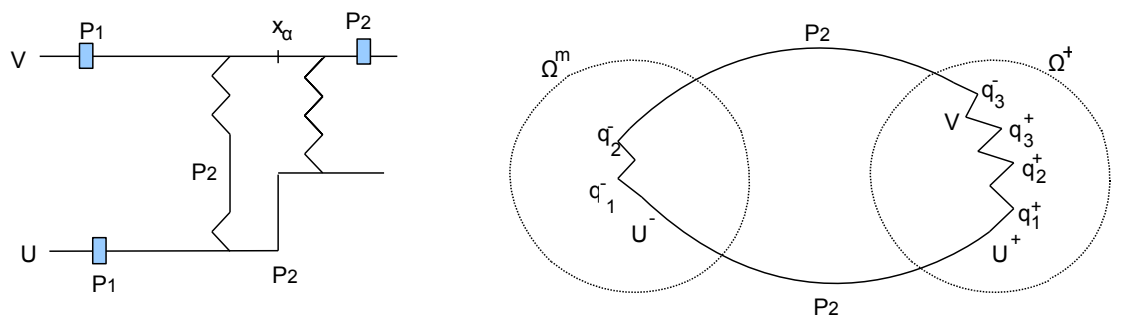

Figure 2.5.5. Case 3
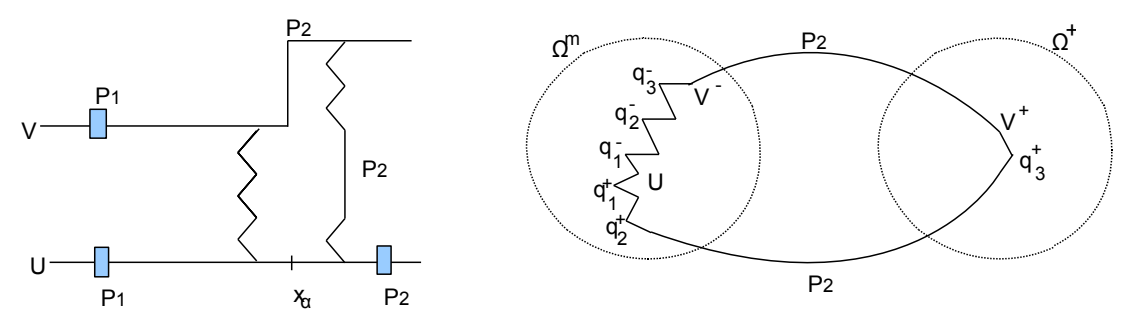

Figure 2.5.6. Case 4

This case is similar to Case 1. (2.4.9) holds if we choose $\kappa_{1}$ and the weights $\tilde{w}_{1}^{+}, \tilde{w}_{2}^{+}$large enough.

Case 4 See Fig 2.5.6.

This case is similar to Case 2. If we choose $\kappa_{1}$ large and the weight $\tilde{w}_{3}^{+}$small enough, (2.4.9) holds under the Stability Condition.

2.5.2. Cases of Small Physical Waves-The Estimate (2.4.10). We consider the cases where the discontinuities in $U$ or $V$ are physical waves. (2.4.10) can be obtained in the same way as [8], if both $U$ and $V$ lie in the same region, stable or metastable, near $x=x_{\alpha}$. Such that, under the assumption that a phase boundary moves slower than any 1 or 3 - waves, we only need to consider the following four cases.

Case A See Fig2.5.7. 

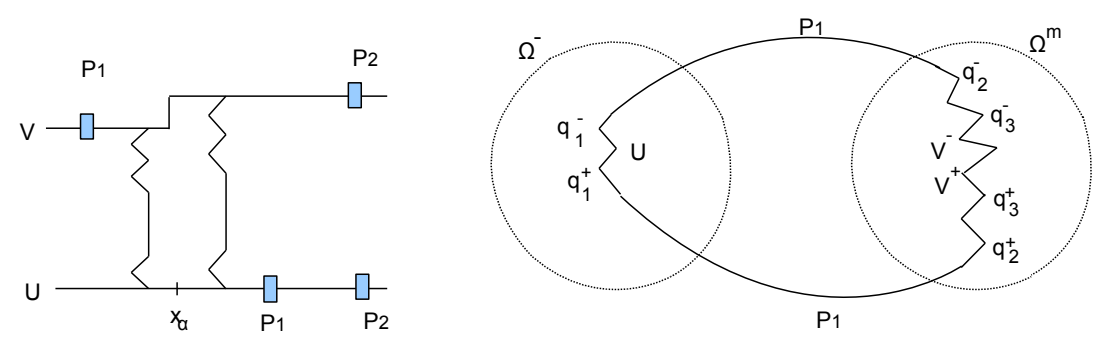

Figure 2.5.7. Case A

When $k_{\alpha}=1$, we have

$$
\begin{aligned}
E_{1 \frac{1}{2}}= & \left|q_{\frac{1}{2}}^{+}\right|\left(W_{1 \frac{1}{2}}^{+}-W_{1 \frac{1}{2}}^{-}\right)\left(\lambda_{1 \frac{1}{2}}^{+}-\dot{x}_{\alpha}\right) \\
& +W_{1 \frac{1}{2}}^{-}\left[\left|q_{1 \frac{1}{2}}^{+}\right|\left(\lambda_{1 \frac{1}{2}}^{+}-\dot{x}_{\alpha}\right)-\left|q_{1 \frac{1}{2}}^{-}\right|\left(\lambda_{1 \frac{1}{2}}^{-}-\dot{x}_{\alpha}\right)\right] \\
\leq & D\left(-\kappa_{1}\left|\varepsilon_{\alpha}\right|\right)\left|\lambda_{1 \frac{1}{2}}^{+}-\dot{x}_{\alpha}\right|+O(1)\left|\lambda_{1 \frac{1}{2}}^{+}-\lambda_{1 \frac{1}{2}}^{-}\right| \\
\leq & -\kappa_{1} D \cdot\left|\varepsilon_{\alpha}\right| \cdot\left|\lambda_{1 \frac{1}{2}}^{+}-\dot{x}_{\alpha}\right|+O(1)\left|\varepsilon_{\alpha}\right| .
\end{aligned}
$$

The estimation of $E_{1}$ can be divided into two cases. If $q_{1}^{-} q_{1}^{+}>0$, we have

$$
\begin{aligned}
= & \left|q_{1}^{ \pm}\right|\left(W_{1}^{+}-W_{1}^{-}\right)\left(\lambda_{1}^{ \pm}-\dot{x}_{\alpha}\right) \\
& +W_{1}^{\mp}\left[\left|q_{1}^{+}\right|\left(\lambda_{1}^{+}-\dot{x}_{\alpha}\right)-\left|q_{1}^{-}\right|\left(\lambda_{1}^{-}-\dot{x}_{\alpha}\right)\right] \\
\leq & -\left|q_{1}^{ \pm}\right| \cdot \kappa_{1}\left|\varepsilon_{\alpha}\right| \cdot\left|\lambda_{1}^{ \pm}-\dot{x}_{\alpha}\right| \\
& +\left(O(1)+\kappa_{1} D\right)\left[\left(\left|q_{1}^{+}\right|-\left|q_{1}^{-}\right|\right)\left(\lambda_{1}^{+}-\dot{x}_{\alpha}\right)+\left|q_{1}^{-}\right|\left(\lambda_{1}^{+}-\lambda_{1}^{-}\right)\right] \\
\leq & \left(O(1)+\kappa_{1} D\right)\left[O(1)\left|q_{1}^{+}-q_{1}^{-}\right|+O(1)\left|q_{1}^{-}\right| \cdot\left|\varepsilon_{\alpha}\right|\right] .
\end{aligned}
$$

Otherwise, if $q_{1}^{-} q_{1}^{+}<0$, then $W_{1}^{+}=W_{1}^{-}$and

$$
E_{1} \leq\left(O(1)+\kappa_{1} D\right)\left[O(1)\left|q_{1}^{+}-q_{1}^{-}\right|+O(1)\left|q_{1}^{-}\right| \cdot\left|\varepsilon_{\alpha}\right|\right] .
$$

For $E_{2}$, we have

$$
\begin{aligned}
E_{2} & =\left[\left|q_{2}^{-}\right|\left(W_{2}^{-}-W_{2}^{+}\right)+W_{2}^{+}\left(\left|q_{2}^{-}\right|-\left|q_{2}^{+}\right|\right)\right] \dot{x}_{\alpha} \\
& \leq-\kappa_{1}\left|\varepsilon_{\alpha}\right| \cdot\left|q_{2}^{-}\right| \cdot\left|\dot{x}_{\alpha}\right|+\left(O(1)+\kappa_{1} D\right) \cdot\left|q_{2}^{+}-q_{2}^{-}\right| \cdot\left|\dot{x}_{\alpha}\right| \\
& \leq\left(O(1)+\kappa_{1} D\right) \cdot\left|q_{2}^{+}-q_{2}^{-}\right| \cdot\left|\dot{x}_{\alpha}\right| .
\end{aligned}
$$

In addition, as $W_{3}^{+}=W_{3}^{-}$, we have

$$
\begin{aligned}
= & \left|q_{3}^{+}\right|\left(W_{3}^{+}-W_{3}^{-}\right)\left(\lambda_{3}^{+}-\dot{x}_{\alpha}\right) \\
& +W_{3}^{-}\left[\left|q_{3}^{+}\right|\left(\lambda_{3}^{+}-\dot{x}_{\alpha}\right)-\left|q_{3}^{-}\right|\left(\lambda_{3}^{-}-\dot{x}_{\alpha}\right)\right] \\
\leq & \left(O(1)+\kappa_{1} D\right)\left[\left(\left|q_{3}^{+}\right|-\left|q_{3}^{-}\right|\right)\left(\lambda_{3}^{+}-\dot{x}_{\alpha}\right)+\left|q_{3}^{-}\right|\left(\lambda_{3}^{+}-\lambda_{3}^{-}\right)\right] \\
\leq & \left(O(1)+\kappa_{1} D\right)\left[O(1)\left|q_{3}^{+}-q_{3}^{-}\right|+O(1)\left|q_{3}^{-}\right| \cdot\left|\varepsilon_{\alpha}\right|\right] .
\end{aligned}
$$


Summing (2.5.11), (2.5.12) (or (2.5.13)), (2.5.14) and (2.5.15) gives

$$
\begin{aligned}
\sum_{k=1,1 \frac{1}{2}, 2,3} E_{k} \leq & -\kappa_{1} D \cdot\left|\varepsilon_{\alpha}\right| \cdot\left|\lambda_{1 \frac{1}{2}}^{+}-\dot{x}_{\alpha}\right|+O(1)\left|\varepsilon_{\alpha}\right|+O(1)\left(1+\kappa_{1} D\right) \\
& \cdot\left[\left|q_{1}^{+}-q_{1}^{-}\right|+\left|q_{2}^{+}-q_{2}^{-}\right|+\left|q_{3}^{+}-q_{3}^{-}\right|+\left(\left|q_{1}^{-}\right|+\left|q_{3}^{-}\right|\right)\left|\varepsilon_{\alpha}\right|\right] .
\end{aligned}
$$

Such that (2.4.9) holds if we choose $\kappa_{1}$ large enough and all the weights $\tilde{w}_{k}^{ \pm}$very small. (Notice that $\left|\varepsilon_{\alpha}\right|$ will not be changed.)

When $k_{\alpha}=2, \dot{x}_{\alpha}=0$. Hence

$$
\begin{aligned}
E_{1 \frac{1}{2}} & =\left|q_{\frac{1}{2}}^{+}\right| W_{1 \frac{1}{2}}^{+} \lambda_{1 \frac{1}{2}}^{+}-\left|q_{1 \frac{1}{2}}^{-}\right| W_{1 \frac{1}{2}}^{-} \lambda_{1 \frac{1}{2}}^{-} \\
& =D\left[\left(W_{1 \frac{1}{2}}^{+}-W_{1 \frac{1}{2}}^{-}\right) \lambda_{1 \frac{1}{2}}^{+}+W_{1 \frac{1}{2}}^{-}\left(\lambda_{1 \frac{1}{2}}^{+}-\lambda_{1 \frac{1}{2}}^{-}\right)\right] \\
& \leq-D \kappa_{1}\left|\varepsilon_{\alpha}\right| \cdot\left|\sigma_{P_{1}}\right|+O(1) D\left|\varepsilon_{\alpha}\right|
\end{aligned}
$$

and

$$
\begin{aligned}
E_{1}= & \left|q_{1}^{+}\right|\left(W_{1}^{+}-W_{1}^{-}\right) \lambda_{1}^{+}+W_{1}^{-}\left[\left|q_{1}^{+}\right| \lambda_{1}^{+}-\left|q_{1}^{-}\right| \lambda_{1}^{-}\right] \\
\leq & -\left|q_{1}^{+}\right| \cdot \kappa_{1}\left|\varepsilon_{\alpha}\right| \cdot\left|\lambda_{1}^{+}\right|+\left(O(1)+\kappa_{1} D\right) \\
& \cdot\left[\left(\left|q_{1}^{+}\right|-\left|q_{1}^{-}\right|\right) \lambda_{1}^{+}+\left|q_{1}^{-}\right|\left(\lambda_{1}^{+}-\lambda_{1}^{-}\right)\right] \\
\leq & \left(O(1)+\kappa_{1} D\right)\left[O(1)\left|q_{1}^{+}-q_{1}^{-}\right|+O(1)\left|q_{1}^{-}\right| \cdot\left|\varepsilon_{\alpha}\right|\right] .
\end{aligned}
$$

We can see by the definition that $E_{2}=0$. Moreover,

$$
\begin{aligned}
E_{3}= & \left|q_{3}^{+}\right|\left(W_{3}^{+}-W_{3}^{-}\right) \lambda_{3}^{+}+W_{3}^{-}\left[\left|q_{3}^{+}\right| \lambda_{3}^{+}-\left|q_{3}^{-}\right| \lambda_{3}^{-}\right] \\
\leq & -\kappa_{1}\left|\varepsilon_{\alpha}\right| \cdot\left|q_{3}^{+}\right| \cdot\left|\lambda_{3}^{+}\right|+\left(O(1)+\kappa_{1} D\right) \\
& \cdot\left[\left(\left|q_{3}^{+}\right|-\left|q_{3}^{-}\right|\right)\left(\lambda_{3}^{+}+\left|q_{3}^{-}\right|\left(\lambda_{3}^{+}-\lambda_{3}^{-}\right)\right]\right. \\
\leq & \left(O(1)+\kappa_{1} D\right)\left[O(1)\left|q_{3}^{+}-q_{3}^{-}\right|+O(1)\left|q_{3}^{-}\right| \cdot\left|\varepsilon_{\alpha}\right|\right] .
\end{aligned}
$$

Summing (2.5.16), (2.5.17) and (2.5.18) gives

$$
\begin{aligned}
\sum_{k=1,1 \frac{1}{2}, 2,3} E_{k} \leq & -D \kappa_{1}\left|\varepsilon_{\alpha}\right| \cdot\left|\sigma_{P_{1}}\right|+O(1) D\left|\varepsilon_{\alpha}\right|+\left(O(1)+\kappa_{1} D\right) \\
& \cdot\left[O(1)\left|q_{1}^{+}-q_{1}^{-}\right|+O(1)\left|q_{3}^{+}-q_{3}^{-}\right|+O(1)\left|q_{1}^{-}\right| \cdot\left|\varepsilon_{\alpha}\right|\right]
\end{aligned}
$$

and (2.4.9) holds if we choose $\kappa_{1}$ large and all the weights $\tilde{w}_{k}^{ \pm}$small enough.

The case where $k_{\alpha}=3$ is similar to that where $k_{\alpha}=1$. We have

$$
E_{1 \frac{1}{2}} \leq-\kappa_{1} D \cdot\left|\varepsilon_{\alpha}\right| \cdot\left|\lambda_{1 \frac{1}{2}}^{+}-\dot{x}_{\alpha}\right|+O(1)\left|\varepsilon_{\alpha}\right| .
$$

Since $W_{1}^{+}=W_{1}^{-}$,

We have

$$
E_{1} \leq\left(O(1)+\kappa_{1} D\right)\left[O(1)\left|q_{1}^{+}-q_{1}^{-}\right|+O(1)\left|q_{1}^{-}\right| \cdot\left|\varepsilon_{\alpha}\right|\right] .
$$

and

$$
E_{2} \leq\left(O(1)+\kappa_{1} D\right) \cdot\left|q_{2}^{+}-q_{2}^{-}\right| \cdot\left|\dot{x}_{\alpha}\right|
$$

Thus,

$$
E_{3} \leq\left(O(1)+3 \kappa_{1} D\right)\left[O(1)\left|q_{3}^{+}-q_{3}^{-}\right|+O(1)\left|q_{3}^{-}\right| \cdot\left|\varepsilon_{\alpha}\right|\right] .
$$



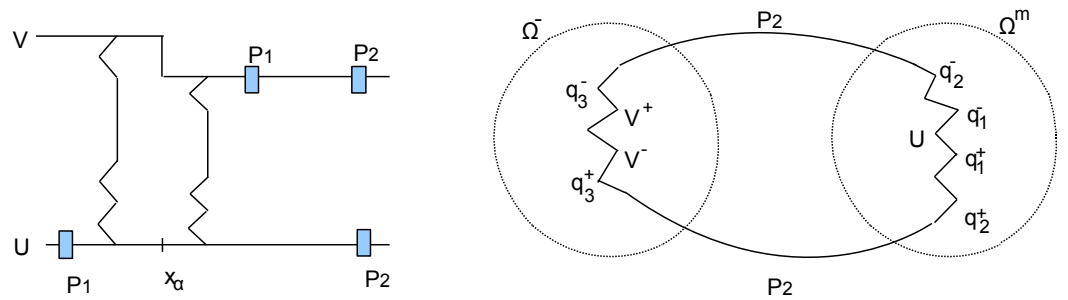

Figure 2.5.8. Case B

$$
\begin{aligned}
\sum_{k=1,1 \frac{1}{2}, 2,3} E_{k} \leq & -\kappa_{1} D \cdot\left|\varepsilon_{\alpha}\right| \cdot\left|\lambda_{1 \frac{1}{2}}^{+}-\dot{x}_{\alpha}\right|+O(1)\left|\varepsilon_{\alpha}\right|+O(1)\left(1+\kappa_{1} D\right) \\
& \cdot\left[\left|q_{1}^{+}-q_{1}^{-}\right|+\left|q_{2}^{+}-q_{2}^{-}\right|+\left|q_{3}^{+}-q_{3}^{-}\right|+\left(\left|q_{1}^{-}\right|+\left|q_{3}^{-}\right|\right)\left|\varepsilon_{\alpha}\right|\right] .
\end{aligned}
$$

Also (2.4.9) holds if we choose $\kappa_{1}$ large and all the weights $\tilde{w}_{k}^{ \pm}$small enough.

\section{Case B See Fig2.5.8.}

In this case, we have $U \in \Omega^{m}$ which is stable and $V \in \Omega^{-}$which is metastable, such that the solution to the Riemann problem $(U, V)$ contains a forward phase boundary $P_{2}$. The analysis of this case is similar to Case A with the large negative term given by $E_{2 \frac{1}{2}}$ instead of $E_{1 \frac{1}{2}}$. We also have (2.4.10) by choosing all the weights $\tilde{w}_{k}^{ \pm}$small enough.

\section{Case C See Fig2.5.9.}

As $U \in \Omega^{+}$is metastable and $V \in \Omega^{m}$ is stable, the solution to the Riemann problem $(U, V)$ contains a backward phase boundary. Therefore, this case is similar to Case A.

Case D See Fig2.5.10.

This case is similar to Case $\mathrm{B}$ and the large negative term is given by $E_{2 \frac{1}{2}}$. 

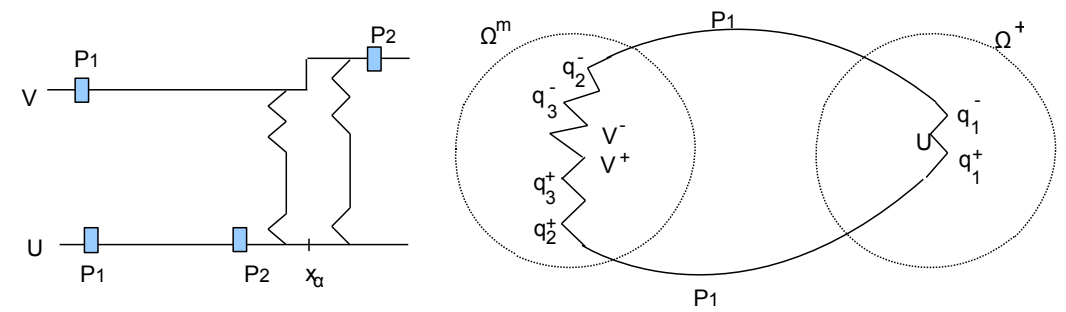

Figure 2.5.9. Case C
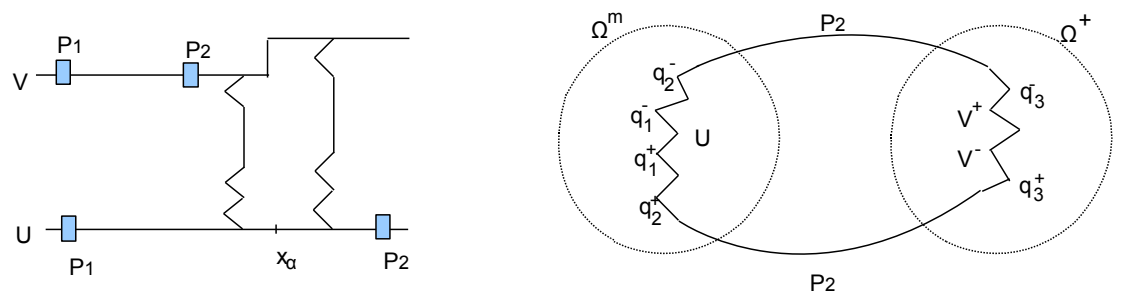

Figure 2.5.10. Case D 


\section{Bibliography}

[1] R. Abeyaratne, J. K. Knowles, Kinetic relations and the propagation of phase boundaries in solids, Arch. Rat. Mech. Anal. 114 (1991), 119-154.

[2] R. Abeyaratne, J. K. Knowles, Dynamics of propagating phase boundaries: thermoelastic solids with heat conduction, Arch. Rational Mech. Anal. 126 (1994) 203-230.

[3] R. Abeyaratne, J. K. Knowles, Dynamics of propagating phase boundaries:adiabatic theory for thermoelastic solids, Physica D. 79 (1994), 269-288.

[4] R. Abeyaratne, J. K. Knowles, Evolution of Phase Transitions, Cambridge University Press, 2006

[5] P. Baiti and H. K. Jenssen, On the front tracking algorithm, J. Math. Anal. Appl. 217 (1998), 395-404.

[6] A. Bressan, Global solutions of systems of conservation laws by wave front tracking, J. Math. Anal. Appl. 170 (1992), 414-432.

[7] A. Bressan, G. Crasta, and B. Piccoli, Well posedness of the Cauchy problem for $n \times n$ conservation laws, Mem. Amer. Math. Soc. 146 (2000), 694.

[8] A. Bressan, T. P. Liu, and T. Yang, $L^{1}$ Stability estimates for $n \times n$ conservation laws, Arch. Rational Mech. Anal. 149 (1999), 1-22.

[9] E. Fermi, Thermodynamics, Dover Publications Inc., 1956.

[10] J. Glimm, Solutions in the large for nonlinear hyperbolic systems of equations, Comm. Pure Appl. Math., 18 (1965), 95-105.

[11] H. Hattori, The Riemann problem for thermoelastic materials with phase change, J. Differential Equ. 205 (2004) 229-252.

[12] H. Hattori, Existence of solutions with moving phase boundaries in thermoelasticity, J. Hyperbolic Differ. Equ. 5(2008), no. 3, 598-611.

[13] P.G. LeFloch, Propagating phase boundaries: Formulation of the problem and existence via Glimm's scheme, Arch. Rational Mech. Anal. 123 (1993) 153-197.

[14] R. J. LeVeque, Finite Volume Methods for Hyperbolic Problems, Cambridge University Press, 2002.

[15] M. Lewicka, $L^{1}$ stability of patterns of non-interacting large shock waves, Indiana Univ. Math. J. 49 (2000) 1515-1537.

[16] M. Lewicka, Stability conditions for patterns of non-interacting large shock waves, SIAM J. Math. Anal. 32 No.5 (2001) 1094-1116.

[17] M. Lewicka and K. Trivisa, On the $L^{1}$ well posedness of systems of conservation laws near solutions containing two large shocks, J. Diff. Eqns. 179 (2002) 133-177.

[18] T. P. Liu and T. Yang, $L^{1}$ Stability of conservation laws with coinciding Hugoniot and characteristic curves, Indiana Univ. Math. J. 48, No. 1 (1999), 237-247.

[19] T. P. Liu and T. Yang, $L^{1}$ Stability of weak solutions for $2 \times 2$ systems of hyperbolic conservation laws, J. Amer. Math. Soc. 12 (1999), 729-774.

[20] The MathWorks, Inc.

[21] S.-C. Ngan, Lev Truskinovsky, Thermal trapping and kinetics of martensitic phase boundaries, J. Mechanics and Physics of Solids, 47(1999), 141-172.

[22] J. Smoller, Shock Waves and Reaction-Diffusion Equations, Springer-Verlag New York Inc., 1983.

[23] A. Vainchtein, Dynamics of Non-isothermal Martensitic Phase Transitions and Hysteresis, Inter. J. of Solids and Structures 39, (2002) 3387-3408 